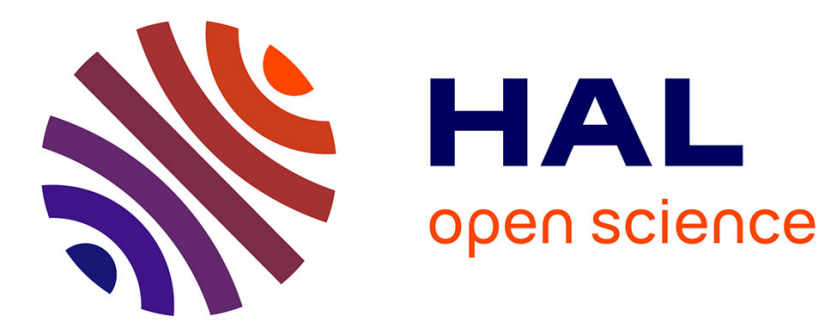

\title{
Analysis of subprotonospheric whistlers observed by DEMETER: A case study
}

Jaroslav Chum, Ondřej Santolík, Michel Parrot

\section{To cite this version:}

Jaroslav Chum, Ondřej Santolík, Michel Parrot. Analysis of subprotonospheric whistlers observed by DEMETER: A case study. Journal of Geophysical Research Space Physics, 2009, 114 (A2), pp.n/an/a. 10.1029/2008JA013585 . insu-03218340

\section{HAL Id: insu-03218340 \\ https://hal-insu.archives-ouvertes.fr/insu-03218340}

Submitted on 5 May 2021

HAL is a multi-disciplinary open access archive for the deposit and dissemination of scientific research documents, whether they are published or not. The documents may come from teaching and research institutions in France or abroad, or from public or private research centers.
L'archive ouverte pluridisciplinaire HAL, est destinée au dépôt et à la diffusion de documents scientifiques de niveau recherche, publiés ou non, émanant des établissements d'enseignement et de recherche français ou étrangers, des laboratoires publics ou privés. 


\title{
Analysis of subprotonospheric whistlers observed by DEMETER: A case study
}

\author{
Jaroslav Chum, ${ }^{1}$ Ondrej Santolik, ${ }^{2,3}$ and Michel Parrot ${ }^{4}$ \\ Received 7 July 2008; revised 31 October 2008; accepted 2 December 2008; published 13 February 2009.
}

[1] Subprotonospheric (SP) whistlers consist of a series of low-dispersion components that result from repeated reflections between the base of the ionosphere and altitudes up to $\sim 1000 \mathrm{~km}$. We have used wave-normal angles and plasma characteristics measured by the DEMETER microsatellite as an input for a three-dimensional ray tracing technique. For several SP whistlers we have also succeeded in finding the causative lightning located at relatively large distances from the satellite footprint along the geomagnetic field line. We show that the reflections and formation of the SP whistlers take place owing to an oblique propagation, with respect to the magnetic field, in the waveguide formed by a profile of the increasing lower hybrid resonance frequency in the upper ionosphere and the base of the ionosphere. We have observed propagation across the magnetic meridian planes. We conclude that the individual components of the SP whistler propagate along different raypaths. The reflected components enter the ionosphere at relatively large distances from the satellite footprint and experience a spread of wave-normal angles during this entry. Depending on the initial wave-normal angle, these waves undergo a different number of reflections before reaching the satellite, thus arriving with different time delays. However, the first component observed of a SP whistler is formed by waves entering the ionosphere at relatively small distances from the satellite footprint and at relatively small wave-normal angles. These waves do not reflect above the satellite but propagate to the opposite hemisphere.

Citation: Chum, J., O. Santolik, and M. Parrot (2009), Analysis of subprotonospheric whistlers observed by DEMETER: A case study, J. Geophys. Res., 114, A02307, doi:10.1029/2008JA013585.

\section{Introduction}

[2] This paper deals with a special class of lightninginduced whistlers called subprotonospheric whistlers (SP whistlers). This name originates from the fact that they appear below the protonosphere, which is a region of the plasmasphere where the protons dominate the other ion species. Thus, their observation is restricted to the altitudes from $\sim 100 \mathrm{~km}$ to $\sim 1000 \mathrm{~km}$ [Carpenter et al., 1964]. The SP whistlers consist of a series of low-dispersion whistler components (echoes); the first component from the series corresponds to a fractional hop whistler, directly upgoing waves. It was suggested that the series of components (echoes) results from repeated reflections between the base of the ionosphere and the protonosphere [Smith, 1964; Kimura, 1966; Raghuram, 1975].

\footnotetext{
${ }^{1}$ Department of Upper Atmosphere, Institute of Atmospheric Physics, Prague, Czech Republic.

${ }^{2}$ Department of Space Physics, Institute of Atmospheric Physics, Prague, Czech Republic.

${ }^{3}$ Also at Faculty of Mathematics and Physics, Charles University, Prague, Czech Republic.

${ }^{4}$ Laboratoire de Physique et Chimie de 1'Environnement, Centre National de la Recherche Scientifique, Orleans, France.

Copyright 2009 by the American Geophysical Union. 0148-0227/09/2008JA013585
}

[3] An example of the SP whistler, observed by Alouette satellite, was first reported by Barrington and Belrose [1963]. Carpenter et al. [1964] performed a statistical analysis. They used data from Alouette satellite records, Aerobee rocket, and ground-based measurements. Alouette observed odd-numbered components at $\sim 1000 \mathrm{~km}$, whereas the rocket and ground station recorded even-numbered hops. The ground observations indicated that the lower reflecting layer was leaky. They found that SP whistlers occur mostly at night, typically within a few hours after the sunset and in most cases at dipole latitudes larger than $45^{\circ}$. The ground observations showed much lower rate of occurrence, probably because of the strong blanketing effect of the ionosphere. They concluded that the mechanism of the lower reflection can probably be explained as a reflection from the sporadic $E$ layer or as a reflection from the lower boundary of the ionosphere. Smith [1964], in a companion paper, presented an explanation of the upper reflection. He put forward the idea that the upper reflection is in fact a refraction phenomenon caused by the change of wave refractive index along the wave trajectories; the waves become highly oblique and propagate across the magnetic field lines in the region of "reflection". Kimura [1966], using the ray tracing technique and diffusive equilibrium model of the upper ionosphere, showed that indeed, the wave of frequencies from $\sim 0.7$ to $\sim 2.5 \mathrm{kHz}$ are refracted back toward Earth if the wave vector is sufficiently deviated 
from vertical direction at the peak of $F_{2}$ layer. He suggested that this deviation can occur as a result of horizontal gradients in the ionosphere or as a result of scattering on intense small-scale irregularities in the $E$ layer. He also proposed that the series of echoes (components) are formed by waves traveling back and forth along one specific path. An alternative interpretation was introduced by Raghuram [1975], who suggested that the multiple traces of an SP whistler are due to wave packets which enter the ionosphere at various latitudes and travel along different paths, exhibiting different number of reflections before reaching the satellite. Egeland and Leer [1970] investigated the cases of simultaneous occurrence of SP whistlers with ion cyclotron whistlers [Gurnett and Shawhan, 1965] using data from OV1-10 satellite. They also presented observation of SP whistlers at latitudes around $20^{\circ}$, which is much lower than reported by Carpenter et al. [1964].

[4] Gurnett et al. [1971] measured the Poynting flux on the Injun 5 satellite at an altitude of $\sim 720 \mathrm{~km}$ and experimentally confirmed that SP whistlers are really repeated reflections between the base of the ionosphere and protonosphere. They found that the leading edge of each successive trace was upgoing and the trailing edge was downgoing. That indicated that the satellite was much closer to the upper reflection point than to the lower reflection point. The Poynting flux measurement on Injun 5 employed one electric and one magnetic antenna, both oriented perpendicular to the magnetic field and to each other. Thus, they could only resolve the Poyntig flux direction up or down the geomagnetic field and they were unable to measure wave-normal directions. Gurnett et al. [1971] also mentioned an interesting, unexplained systematic variation of the upper and lower cutoff frequencies of components (echoes). Russell et al. [1972], reviewing the investigations of extremely low frequency (ELF) and very low frequency (VLF) waves in Earth's magnetosphere stated that SP whistlers remained one whistler phenomenon that required further experimental and theoretical study. Nevertheless, since then there has been little activity in studying this interesting phenomenon.

[5] In this paper, we present a detailed wave-normal analysis of SP whistlers observed by the DEMETER satellite launched to a nearly circular quasi Sun-synchronous orbit at the altitude of $\sim 700 \mathrm{~km}$ in 2004 . We perform a ray tracing analysis with the initial values given by this wave-normal analysis and with a diffusive equilibrium model adjusted to observed plasma parameters. This combination of wave-normal measurement on board DEMETER with ray tracing simulation is the same procedure as the one used by Santolik et al. [2009], who investigated propagation of fractional-hop whistlers from the ground. Here we use it to investigate propagation of SP whistlers. For several SP whistlers, we have also succeeded in finding a causative lightning discharge using data provided by the European Cooperation for Lightning Detection (EUCLID). We show that SP whistlers can originate from strong lightning located at relatively large distances in direction perpendicular to the meridian plane. Section 2 shows examples of the SP whistlers observed by DEMETER and results of the wave-normal analysis. Section 3 presents the ray tracing simulations with initial conditions adjusted to the observations. Section 4 discusses the results obtained by the ray tracing simulations and compares them with the observations. Section 5 provides conclusions of our work.

\section{Observations of SP Whistlers and Plane Wave Analysis}

[6] The French microsatellite DEMETER was launched to a nearly circular orbit from Baikonur (Kazakhstan) on 29 June 2004. Its initial altitude of $\sim 710 \mathrm{~km}$ was lowered to $\sim 660 \mathrm{~km}$ at the end of 2005 . The main scientific objective of DEMETER is to study the disturbances of the ionosphere owing to the seismic and volcanic effects and the influence of anthropogenic activities (power line harmonic radiation PLHR, VLF transmitters, HF broadcasting stations) on the ionosphere and radiation belts. Its scientific payload makes also possible the investigation of natural geomagnetic phenomena occurring in the near-Earth plasma environment.

[7] The DEMETER satellite carries two instruments dedicated to the measurements of electromagnetic waves. The ICE experiment [Berthelier et al., 2006a] measures the electric field between four spherical sensors located on booms. The desired component of electric field is chosen by a selection of these sensors. The IMSC experiment [Parrot et al., 2006] is composed of a three-axis search coil magnetometer. The scientific experiments are working in two different modes of operation: a burst mode, which is mainly activated above seismic zones [Parrot et al., 2006], and a survey mode used elsewhere. In the survey mode, only the onboard calculated spectra with the time resolution of $2.048 \mathrm{~s}$ or $0.512 \mathrm{~s}$ are recorded. This is insufficient for the detection and analysis of SP whistlers. Therefore, waveforms recorded in the burst mode are used in our work. The full vectors of the electromagnetic field, i.e., three components of electric and three components of magnetic field, are only available in the frequency range up to $\sim 1 \mathrm{kHz}$ (sampling frequency $2.5 \mathrm{kHz}$ ). In the VLF range (up to $\sim 17 \mathrm{kHz}$ with sampling frequency $40 \mathrm{kHz}$ ), the waveforms of one electric and one magnetic component are recorded.

[8] Figure 1 presents a $20 \mathrm{~s}$ length spectrogram computed from the waveform of electric field recorded at magnetic latitude $50.3^{\circ}$ and $\sim 22.6$ magnetic local time on 9 July 2007 from 2107:22.2 UT to 2107:42.2. Several SP whistlers can be clearly recognized near $\sim 1 \mathrm{kHz}$ in this spectrogram. An intense and clear SP whistler occurred at $\sim 2107: 26.3(\sim 4 \mathrm{~s}$ after the beginning of the spectrogram). We selected this SP whistler as a characteristic example used for our study. Note also the low hybrid resonance (LHR) emissions around the frequency of $\sim 12 \mathrm{kHz}$ induced probably by diffusive whistlers propagating from the opposite hemisphere. These emissions will be shortly discussed in the section 4.2.

[9] Figure 2 shows results of the multicomponent wave analysis performed under the approximation of a plane wave on the basis of the singular value decomposition (SVD) method [Santolik at al., 2003, 2006a, 2009] in the short time interval from 2107:26 UT to 2107:29 containing the intense SP whistler. The orientation of the $x, y, z$ axes of the Cartesian coordinate system used in this plane wave analysis is as follows: the $z$ axis is directed along the magnetic field lines, the $x$ axis lies in the magnetic meridian plane and is directed from Earth. We will focus in our 


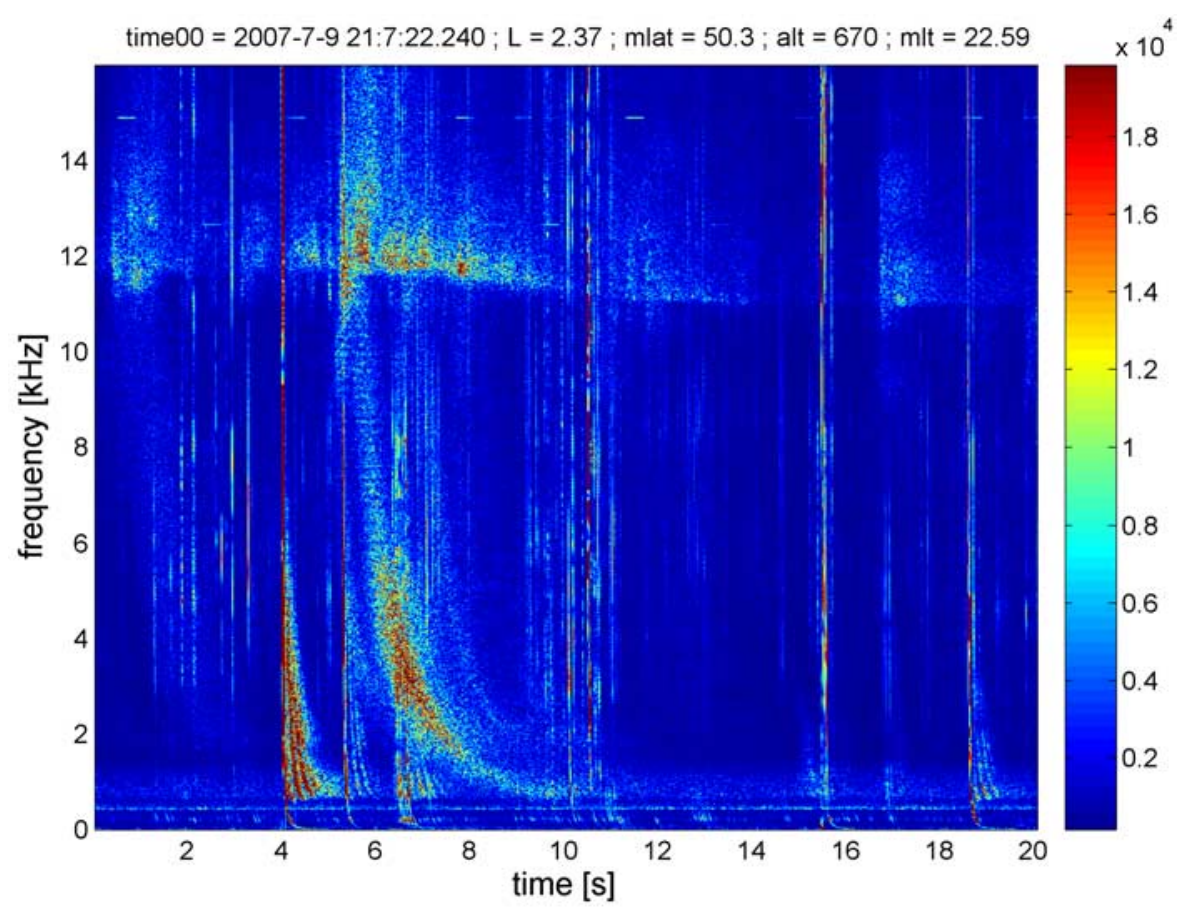

Figure 1. Amplitude spectrogram computed from the burst mode of the VLF data recorded by ICE on board DEMETER on 9 July 2007 from 2107:22.2 to 2107:42.2 UT. The orbital parameters are related to the beginning of the spectrogram. The color-coded (gray scale) spectral density of the signal amplitude is given in $\mu \mathrm{V} / \mathrm{m} \mathrm{Hz}^{-1 / 2}$.

discussion on the intense SP whistler observed from $\sim 2107: 26.3$ to $\sim 2107: 26.9$. The numbers displayed in brackets in each panel indicate the individual components of the SP whistler analyzed. The two top panels display the power spectrograms of the wave magnetic (Figure 2a) and electric field (Figure 2b), respectively. It is obvious that the subsequent components are stronger in the electric field than in the magnetic field. The ellipticity, shown in Figure 3c, indicates that the first trace (component) formed by the upgoing waves of the fractional hop whistler $(0+$ whistler $)$ corresponds to the right-handed waves with nearly circular polarization, whereas the subsequent components are highly elliptical with nearly linear polarization. The ellipticity is +1 (red color) for right-handed circular waves, -1 (blue color) for left-handed circular waves, and close to 0 for linearly polarized waves. Worth noticing is also the left-handed proton whistler [Gurnett and Shawhan, 1965] observed just below the proton cyclotron frequency marked by the horizontal line at $\sim 550 \mathrm{~Hz}$. Figures $2 \mathrm{~d}$ and $2 \mathrm{e}$ present the wavenormal angle $\theta$ (polar angle between the magnetic field and wave vector) and the azimuth $\varphi$ of the wave vector in the $x-y$ plane ( 0 corresponds to the wave vector lying in the $x-z$ plane, i.e., directed from Earth, $90^{\circ}$ to the wave vector directed eastward, $180^{\circ}$ to the Earthward direction, and $-90^{\circ}$ to the westward direction). It is obvious that the first trace $(0+$ whistler) propagates nearly antiparallel to the magnetic field lines; the typical values of angles $\theta$ and $\varphi$ are $160^{\circ}$ and $-70^{\circ}$, respectively. A different situation is observed in the case of reflected components (echoes). These are formed by nearly transverse waves having the wave vector almost perpendicular to the magnetic field. This is in agreement with the theory of refraction suggested by Smith [1964] and Kimura [1966]. However, worth noticing is the azimuthal angle $\varphi$ indicating that the wave vector is also almost perpendicular to the meridian plane in this case. Note that Smith and Kimura mainly considered the 2-D case in the meridian plane. The last two panels stand for the $z$ - and $x$-components of the Poynting flux $S_{Z}$ (Figure 2f) and $S_{X}$ (Figure 2g) normalized to their standard deviations. The $S_{Z}$ component is positive (red) if the waves propagate downward along the magnetic field on the Northern Hemisphere, and the $S_{X}$ component is positive for waves propagating northward. From Figure $2 \mathrm{~g}$, it is obvious that the first trace is upgoing, whereas the second trace (component) is formed by the downgoing waves. As for the subsequent components, the leading edges are upgoing and the trailing edges are downgoing, which indicates that these components reflected just above the satellite. These results are similar to those obtained by Gurnett et al. [1971]. In turn, the second trace differs in the $S_{X}$ component. The leading edge propagates northward, whereas the trailing edge corresponds to the waves propagating southward. This different behavior of the second trace is also seen in the azimuthal angle $\varphi$.

[10] As for other whistlers seen in Figure 2, a weak SP whistler was observed before 2107:28. Because of its weakness, it is inconvenient for the analysis. Note that the second and third components are practically invisible in magnetic field. Before 2107:29, multiple 0+ whistlers corresponding to multiple lightning strokes are observed.

[11] Summarizing the results of plane wave analysis presented in Figure 2, we state that the echoes (reflected components) of SP whistler are represented by the highly oblique waves propagating nearly perpendicularly to the 


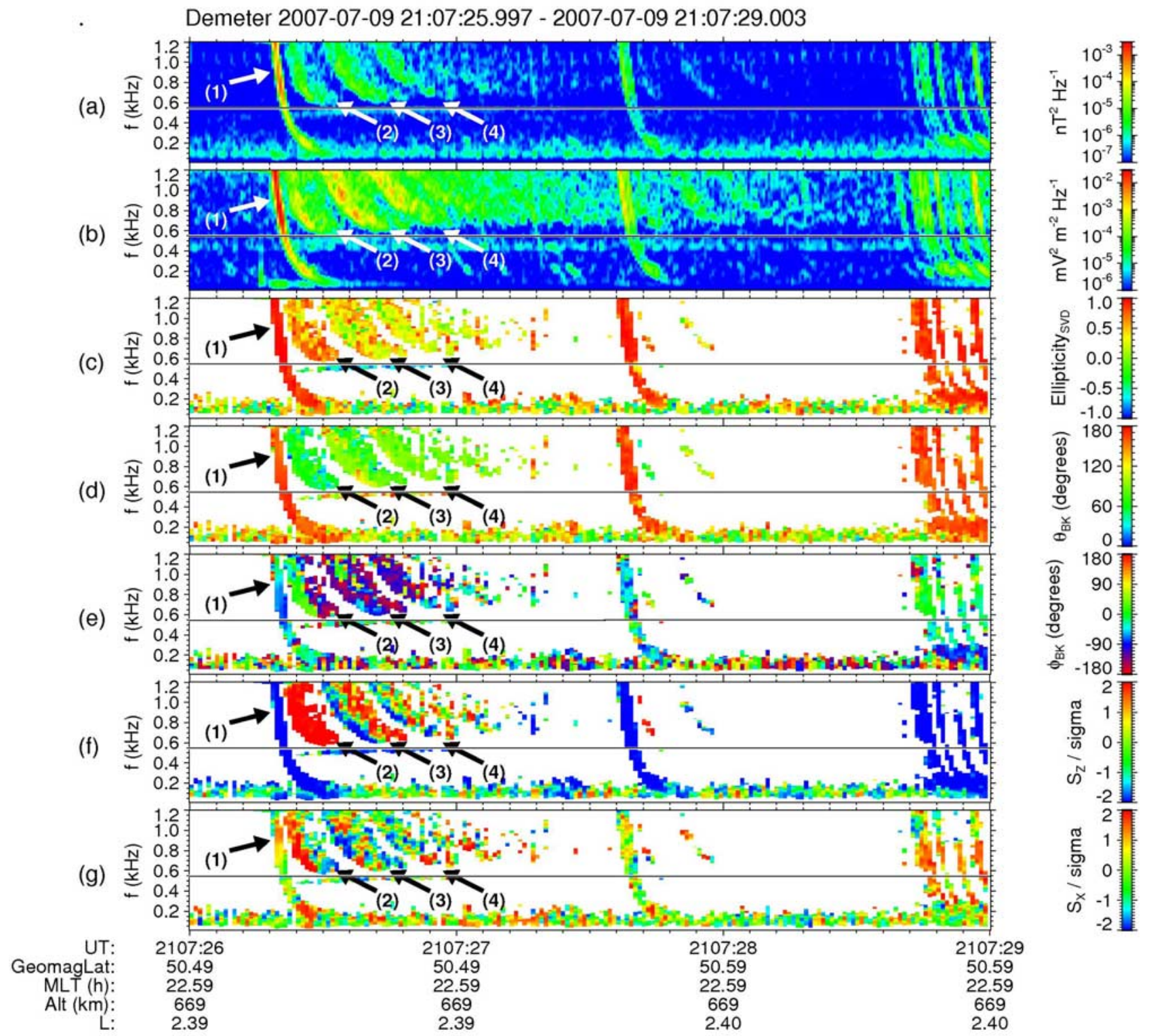

Figure 2. A plane wave analysis of the selected time interval from 2107:26 to 2107:29 UT on 9 July 2007. From top to bottom: (a) power spectrogram of the magnetic field, (b) power spectrogram of the electric field, (c) ellipticity ( 1 for circularly polarized right-handed waves, 0 for linear polarization, and -1 for circularly polarized left-handed waves), (d) polar angle $\theta$ ( 0 for waves propagating parallel to magnetic field and $180^{\circ}$ for waves propagating antiparallel to magnetic field), (e) azimuthal angle $\varphi$ ( 0 for waves propagating outward from Earth in meridian plane, $180^{\circ}$ for waves propagating Earthward in meridian plane, and $90^{\circ}$ for waves propagating eastward, perpendicular to meridian plane), (f) parallel component of the Poynting flux $S_{Z}$ normalized to its standard deviation (red for waves propagating parallel to magnetic field, i.e., downward on the Northern Hemisphere; blue for waves propagating antiparallel to magnetic field, i.e., upward on the Northern Hemisphere), and (g) perpendicular component of the Poynting flux $S_{X}$ along the $x$ axis lying in the meridian plane (red for waves propagating outward from Earth and blue for waves propagating to Earth). The black horizontal lines at $\sim 550 \mathrm{~Hz}$ indicate the measured proton cyclotron frequency.

magnetic field with wave vectors oriented predominantly westward. This finding is consistent with the location of causative lightning. To assign the causative lightning discharge to the SP whistler, we used the data from the European lightning detection network EUCLID and the method on the basis of the statistical approach described by Chum et al. [2006] taking into account a possible small time shift between the clocks used in the EUCLID network and aboard the DEMETER satellite ( $\sim 80 \mathrm{~ms}$ in this case). Table 1 presents characteristics of lightning for which we have found corresponding whistler in the spectrogram presented in Figure 1. The given characteristics of lightning are time of detection, location (geographical latitude and longitude), discharge current, and type of lightning, where 0 

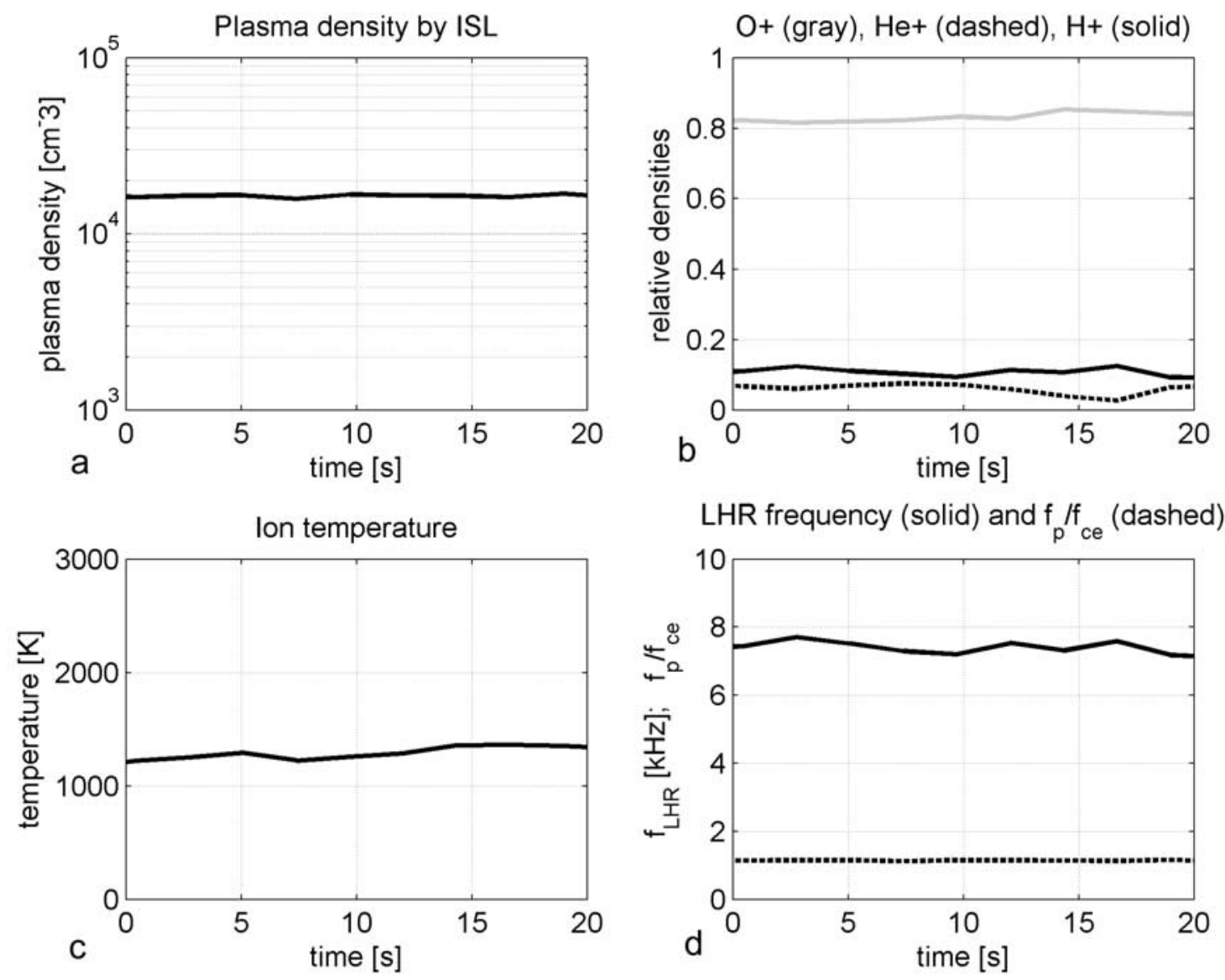

Figure 3. Plasma characteristics in the time interval corresponding to spectrogram in Figure 1. (a) Electron density measured by the Langmuir probe (ISL experiment). (b) Relative ion densities measured by the Retarding Potential Analyzer (IAP experiment). (c) Ion temperature measured by IAP experiment. (d) LHR frequency calculated from the measured values using equation (2) (solid line) and plasma frequency to electron cyclotron frequency ratio (dashed line).

is used for cloud-to-ground discharges (CG) and 1 for intracloud discharges (IC). We also give the coordinates of the DEMETER footprint along the geomagnetic field lines at the base of the ionosphere at the altitude of $110 \mathrm{~km}$ for the lightning to which we found a corresponding SP whistler.
[12] From Table 1, it is obvious that the footprints of the DEMETER satellite were at the latitude of $\sim 52^{\circ}$ and longitude of $\sim 6.8^{\circ}$. The lightning causing the SP whistlers occurred around the latitude of $50^{\circ}$ and longitude $20^{\circ}$. In other words, whereas the latitudes of lightning and the satellite footprints were about the same, the longitudinal

Table 1. Characteristics of Lightning With Whistlers in the Spectrogram Presented in Figure 1 and Satellite Footprint at the Times of the Observations of SP Whistlers ${ }^{\mathrm{a}}$

\begin{tabular}{|c|c|c|c|c|c|}
\hline Time (UT) & $\begin{array}{c}\text { Latitude } \\
\text { (deg) }\end{array}$ & $\begin{array}{c}\text { Longitude } \\
\text { (deg) }\end{array}$ & $\begin{array}{c}\text { Current } \\
(\mathrm{kA})\end{array}$ & Type & DEMETER Footprint at $110 \mathrm{~km}$ \\
\hline $2107: 25.20$ & 49.36 & 19.45 & -9 & 0 & \\
\hline $2107: 26.30$ & 50.39 & 21.58 & 28 & 1 & $\mathrm{SP} ;$ latD $=51.84$, longD $=6.89$ \\
\hline $2107: 27.10$ & 47.6 & 19.59 & -8 & 1 & \\
\hline $2107: 27.60$ & 49.77 & 19.59 & -56 & 0 & $\mathrm{SP} ;$ latD $=51.91$, longD $=6.86$ \\
\hline $2107: 28.70$ & 47.75 & 19.74 & -22 & 0 & \\
\hline $2107: 29.40$ & 50.14 & 21.89 & -15 & 0 & \\
\hline $2107: 36.90$ & 64.02 & 32.65 & -11 & 0 & \\
\hline $2107: 37.80$ & 52.84 & 5.98 & -26 & 1 & \\
\hline $2107: 39.10$ & 45.64 & 9.79 & -10 & 0 & \\
\hline $2107: 39.20$ & 45.62 & 9.93 & 15 & 0 & \\
\hline $2107: 40.00$ & 47.85 & 19.73 & -15 & 0 & \\
\hline $2107: 40.90$ & 49.47 & 19.13 & 72 & 0 & $\mathrm{SP} ;$ latD $=52.65$, longD $=6.52$ \\
\hline $2107: 41.00$ & 49.38 & 18.96 & 21 & 1 & \\
\hline
\end{tabular}

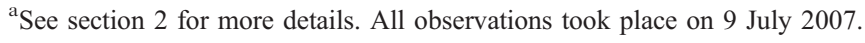


differences were much larger; the satellite footprints were about $13^{\circ}$ westward from the lightning (more than $900 \mathrm{~km}$ ). This topology is in the accordance with the westward propagation of the waves which we obtained by the plane wave analysis. Note also that the SP whistlers resulted from relatively strong discharges. Interestingly, in this case, the first SP whistler was caused by the IC discharge, the second was caused by the negative CG, and the third was caused by the positive $\mathrm{CG}$ discharge. Despite the IC lightning was the weakest of these three, the observed SP whistler was the strongest in this case. We have no explanation for that. We should note that EUCLID facilities are designed to provide best performance in the detection of CG lightning. Only few percent of all IC lightning are detected, and detection efficiency of IC lightning is not uniform over the network area. Note also that we have not succeeded in finding the causative lightning for most of the SP whistlers observed. First, many SP whistlers were observed far from the European region, where we have access to the lightning data. Second, even if we observed SP whistlers above Europe, these SP whistlers could be caused by a lightning which were relatively distant from the satellite footprint as we have shown. Thus, we do not have found enough causative lightning for a statistical study. Actually, we have only found eight causative lightnings to SP whistlers, five of them were positive $\mathrm{CG}$, two were negative $\mathrm{CG}$, and one was IC discharge. In all these cases the causative lightning was relatively strong with respect to the other lightning detected. In one case, the discharge current of the causative lightning was $+167 \mathrm{kA}$.

[13] We have performed the plane wave analysis for seven other SP whistlers. In all these cases, we have found that the second component of SP whistlers was formed by the downgoing waves, whereas the third and next components, if observed, were formed by upgoing and downgoing waves; the upgoing waves prevailed in the leading edges, and the downgoing waves prevailed in trailing edges. The azimuthal wave-normal angle $\varphi$ of these waves indicated that these waves propagated across the meridian plane in most of these cases. Only in one case we observed that the higher components (echoes) propagated approximately in the meridian plane. Therefore, we think that the example selected for our case study represent a typical SP whistler. Our selection was also influenced by the fact that we succeeded in assigning the causative lightning to the SP whistler observed in this case.

[14] The observations of SP whistlers by DEMETER confirm that SP whistlers are mainly nighttime phenomena [Carpenter et al., 1964]. We will discuss this fact in more details in section 4.2. In accord with the study of Carpenter et al. [1964], we have found that the dispersion of the components of the SP whistlers follows rather well the formula $t=D f^{-1 / 2}$ introduced by Eckersley [1935], where $D$ is the dispersion. We will present a brief analysis of the dispersion of the components of SP whistlers in the section 4.4, where we also show that this fact is consistent with the results of the ray tracing simulation. We have not confirmed that the SP whistlers are mainly observed at magnetic latitudes larger than $45^{\circ}$ as was reported by Carpenter et al. [1964]. We have observed the SP whistlers in the range of latitudes from $\sim 30^{\circ}$ to $\sim 57^{\circ}$. However, we have not performed a systematic statistical analysis.
[15] In the next section, we will investigate whether the ray tracing simulations with initial values of wave-normal angles obtained by the multicomponent measurements aboard DEMETER are consistent with the refraction theory of SP whistlers as proposed by Smith [1964] and Kimura [1966].

\section{Ray Tracing Simulations}

[16] We have used ray tracing software described by Santolik et al. [2006b, 2009]. It has been developed with substantial modifications from the original technique of Cerisier [1970] and Cairo and Lefeuvre [1986]. For simplicity, only the dipole approximation of the magnetic field was used in the present study. This is different from the procedure used by Santolik et al. [2009] where the full IGRF model was taken into account. We also note that the diffusive equilibrium plasma density model incorporated in the ray tracing software does not have any gradients in the direction perpendicular to the magnetic meridian plane. Below the maximum of plasma density in $F_{2}$ layer at $\sim 250 \mathrm{~km}$, the diffusive equilibrium model is stopped and the plasma density is supposed to exponentially decrease. The plasma density model was fitted to the plasma measurements carried out on board the DEMETER satellite. We used the electron density measured by the Langmuir probe of the ISL experiment [Lebreton et al., 2006] and ion densities and ion temperature measured by the retarding potential analyzer of the IAP experiment [Berthelier et al., 2006b]. Figure 3 presents the plasma density, ion relative densities, temperature, and LHR frequency in the time interval corresponding to the spectrogram in Figure 1. The LHR frequency $f_{L H}$ was calculated using equation (1) derived for multicomponent plasmas [Smith and Brice, 1964],

$$
\frac{1}{f_{L H}^{2}} \cdot \sum_{i} \frac{A_{i}}{M_{i}}=\frac{1}{f_{p}^{2}}+\frac{1}{f_{c e}^{2}}
$$

where $M_{i}$ is the mass of $i$ th type of ion related to the mass of an electron $m_{e}\left(M_{i}=m_{i} / m_{e}\right), A_{i}$ is the relative density of $i$ th type of ion related to the density of electrons $\left(A_{i}=n_{i} / n_{e}\right), f_{c e}$ is the electron cyclotron frequency $\left(2 \pi f_{c e}=e B / m_{e} ; B\right.$ is the amplitude of magnetic field, $e$ is the charge of an electron), and $f_{p}$ is the plasma frequency defined by equation (2),

$$
\left(2 \pi f_{p}\right)^{2}=\frac{n_{e} e^{2}}{\varepsilon_{0} m_{e}},
$$

where $\varepsilon_{0}$ is the permittivity of the free space.

[17] The plasma characteristics measured by DEMETER and used in the ray tracing simulation were as follows: $n_{e}=$ $16,600 \mathrm{~cm}^{-3}, \mathrm{~T}=1250 \mathrm{~K}, A_{O+}=0.82, A_{\mathrm{He}^{+}}=0.07$, and $A_{H}^{+}=0.11$ at the magnetic latitude mlat $=50.5$ and altitude $667 \mathrm{~km}$. The calculated LHR frequency is $7.73 \mathrm{kHz}$. We start the simulation with the frequency $f=800 \mathrm{~Hz}$ and the typical values of angle $\theta=160^{\circ}$ observed at this frequency for the first trace. The angle $\varphi$ oscillated; its typical values were between $-70^{\circ}$ and $-60^{\circ}$. The ray tracing simulation shows that we do not obtain any reflection for these initial values. The maximum of modeled LHR frequency $f_{L H R}$ calculated along the ray trajectory was $11.04 \mathrm{kHz}$ at the 

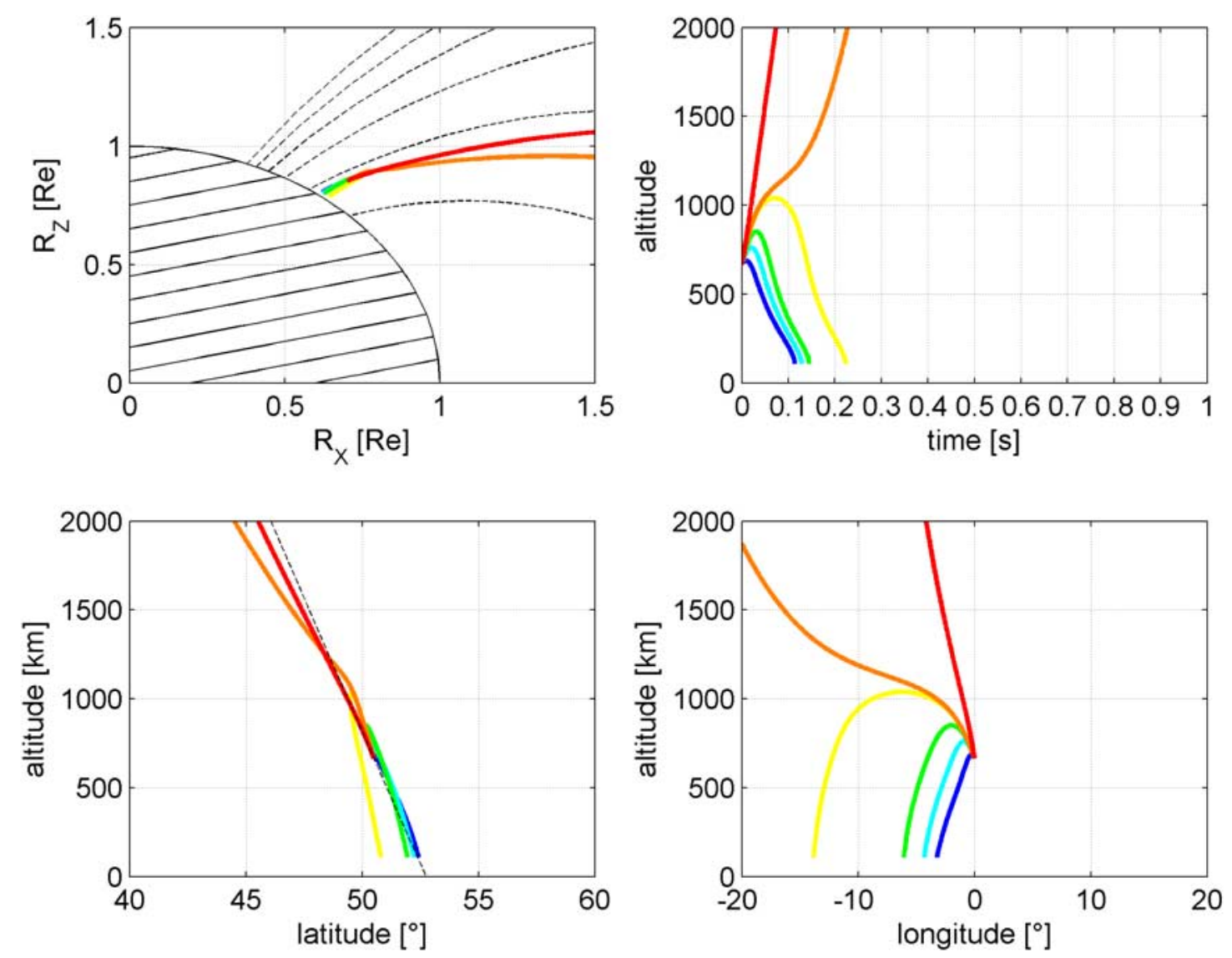

Figure 4. Ray tracing results for raypaths started at the point of the satellite with the angle $\varphi=-70^{\circ}$ and various initial values of $\theta$, which are color-coded as follows: $\theta=95^{\circ}$ (blue), $\theta=105^{\circ}$ (cyan), $\theta=115^{\circ}$ (green), $\theta=124^{\circ}$ (yellow), $\theta=125^{\circ}$ (orange), and $\theta=160^{\circ}$ (red). All coordinates are geomagnetic. The initial longitude of the satellite is supposed to be 0 . Red curves correspond to the first trace. See section 3 for more details.

altitude of $1084 \mathrm{~km}$. In order to get the backward refraction, we have to start the ray tracing simulation with the wavenormal angle $\theta$ less or equal to a critical value of $124^{\circ}$ for the angle $\varphi=-70^{\circ}$. In other words, the rays should be much more deviated from the antiparallel direction at the point of the observation. The results of ray tracing simulation for $\varphi=-70^{\circ}$ and various angles of $\theta$ are presented in Figure 4. The raypaths for various angles $\theta$ are color-coded as follows: $\theta=95^{\circ}$ (blue), $\theta=105^{\circ}$ (cyan), $\theta=115^{\circ}$ (green), $\theta=124^{\circ}$ (yellow), $\theta=125^{\circ}$ (orange), and $\theta=160^{\circ}$ (red). Note that the initial longitude of the satellite is supposed to be 0 in all the ray tracing simulations presented in this article. The results presented in Figure 4 indicate that the more perpendicular is the initial wave vector at the point of the observation; the lower is the altitude at which the rays reflect (the closer the satellite). It is obvious that the results of ray tracing suggest that the main energy of the first trace propagate to the opposite hemisphere and do not refract backward from the region above the satellite. This is similar to the results of Santolik et al. [2009]. We will discuss this fact in more detail later in section 4.

[18] It is also interesting to see how the ray trajectories depend on the azimuthal angle $\varphi$ for a fixed polar angle $\theta$. The results of ray tracing simulations indicate (not shown) that the backward refraction depends also on $\varphi$. The best conditions for the rays to be bent backward are if the wave vector lies in the meridian plane and is directed Earthward, i.e., for $\varphi=-180^{\circ}$. The reflection then occurs for all angles $\theta$ which satisfy the inequality $90^{\circ} \leq \theta \leq 143^{\circ}$. Note that some rays can propagate to significant distances across the meridian planes. This feature is only observed for waves having the wave-normal angles close to the critical values of $\theta$ and $\varphi$ for which the bifurcation of trajectories occurs; i.e., a small change causes that the waves are refracted backward instead of propagating to the opposite hemisphere or vice versa.

[19] Next, we will investigate where the first trace, its main energy respectively, propagated from. We performed the backward ray tracing with the initial values $\theta=20^{\circ}$ and $\varphi=110^{\circ}$. We found that the difference of rays with respect to the satellite footprint is $0.11^{\circ}$ in latitude southward and $0.64^{\circ}$ in longitude eastward at the base of the ionosphere. The wave-normal angles were $\theta=20^{\circ}$ and $\varphi=169.2^{\circ}$ at the base of the ionosphere, which means $\theta=160^{\circ}$ and $\varphi=$ $-10.8^{\circ}$ for the forward propagating rays. The propagation time to the base of the ionosphere was $0.079 \mathrm{~s}$.

[20] As for the second downgoing trace, the typical values are $\theta \sim 65^{\circ}$ and $\varphi \sim 0$ for the leading edge and $\varphi \sim$ $-140^{\circ}$ for the trailing edge, but these values rather fluctuate. We will test whether this trace corresponds to the downward refracted waves by backward ray tracing. 

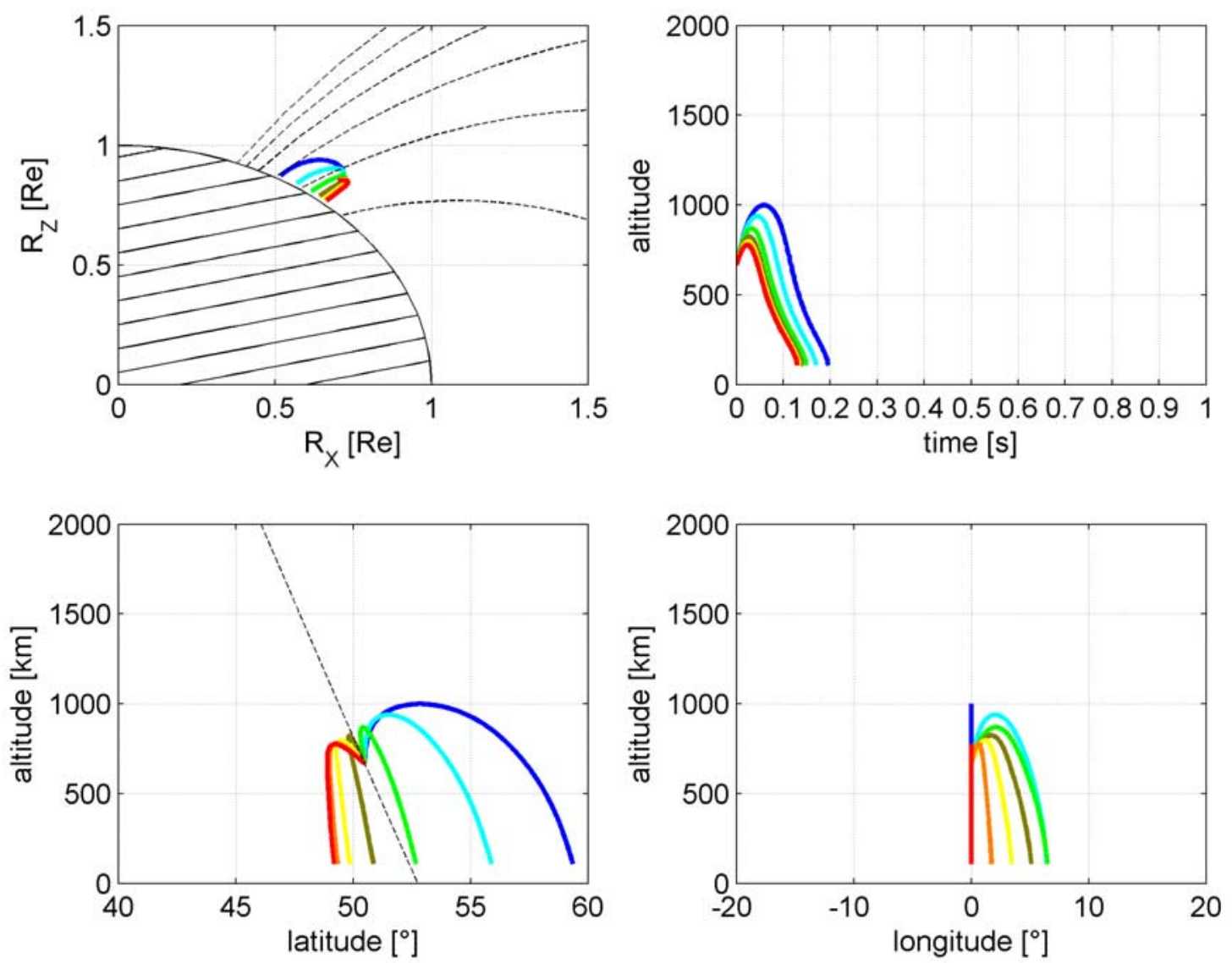

Figure 5. Results of the backward ray tracing for raypaths started at the point of the satellite with the initial angle $\theta=115^{\circ}$ (second trace) and various initial values of $\varphi$, which are color-coded as follows: $\varphi=$ 0 (blue), $\varphi=30^{\circ}$ (cyan), $\varphi=60^{\circ}$ (green), $\varphi=90^{\circ}$ (olive), $\varphi=120^{\circ}$ (yellow), $\varphi=150^{\circ}$ (orange), and $\varphi=$ $180^{\circ}$ (red). All coordinates are geomagnetic. See section 3 for more details.

Figure 5 shows the results of ray tracing simulations with the initial values $\theta=115^{\circ}$ and $\varphi$ varying from 0 to $180^{\circ}$ with the $30^{\circ}$ step. The trajectories are again color-coded. We can see that the altitude of the backward refraction depends on the angle $\varphi$; the smaller the value of $\varphi$, the higher the reflection takes place. The reflection occurred at the altitude of $776 \mathrm{~km}$ for the leading edge, which is well below the maximum of the LHR frequency. The simulated time of propagation to the altitude of the satellite was $0.045 \mathrm{~s}$ and to the base of the ionosphere $0.130 \mathrm{~s}$. The final latitude $\left(49.2^{\circ}\right)$ at the base of the ionosphere is $\sim 2.6^{\circ}$ southward with respect to the satellite footprint. The wave-normal angles are $\theta=39.7^{\circ}$ and $\varphi=180^{\circ}$ at the base of the ionosphere, which means $\theta=140.3^{\circ}$ and $\varphi=0$ for the forward propagating rays. Concerning the trailing edge of the second trace, the simulated backward refraction occurred at the altitude of $913 \mathrm{~km}$. The time of propagation to the altitude of the satellite was $0.079 \mathrm{~s}$ and $0.161 \mathrm{~s}$ to the base of the ionosphere. Note that this longer time of propagation of the trailing edge is consistent with the observation. The difference in latitude and longitude with respect to the satellite footprint at the base of the ionosphere was $\sim 3.8^{\circ}$ northward and $6.8^{\circ}$ eastward, respectively. The wave-normal angles are $\theta=17.2^{\circ}$ and $\varphi=142.1^{\circ}$ at the base of the ionosphere, which means $\theta=162.8^{\circ}$ and $\varphi=-37.9^{\circ}$ for the forward propagating rays.
[21] Next we will investigate the third trace. The measured values are $\theta \sim 96^{\circ}$ and $\varphi \sim-135^{\circ}$ for the leading upgoing edge and $\theta \sim 83^{\circ}$ and $\varphi \sim-120^{\circ}$ for the trailing downgoing edge. We note that there is a relatively large fluctuation in the angle $\varphi$. Figure 6 presents the ray trajectories for $\theta=96^{\circ}$ and $\varphi$ varying from $-60^{\circ}$ to $-180^{\circ}$ with a $20^{\circ}$ step. The trajectories are color-coded as follows: $\varphi=-60^{\circ}$ (blue), $\varphi=-80^{\circ}$ (cyan), $\varphi=-100^{\circ}$ (green), $\varphi=$ $-120^{\circ}$ (olive), $\varphi=-140^{\circ}$ (yellow), and $\varphi=-160^{\circ}$ (orange). Note that the reflections occur at relatively low altitudes for any angle $\varphi$. It is obvious that the waves become more transverse with the number of reflections. The more transverse the waves are, the lower the reflection height is.

[22] Table 2 summarizes some results of the ray tracing simulations for the typical values of angles $\theta$ and $\varphi$ measured by DEMETER at $f=800 \mathrm{~Hz}$ for the first, second, and third traces. The leading and trailing edges of the second and third trace are treated independently. The parameters with the index " 0 " represent the quantities calculated by the backward ray tracing at the base of the ionosphere at the altitude of $110 \mathrm{~km}$; however, the angles are given in the form corresponding to the forward propagating waves. In other words, they correspond to the waves that are going to reach the satellite. The parameters with index " 1 " correspond to the waves calculated by the forward ray tracing at the base of the ionosphere. 

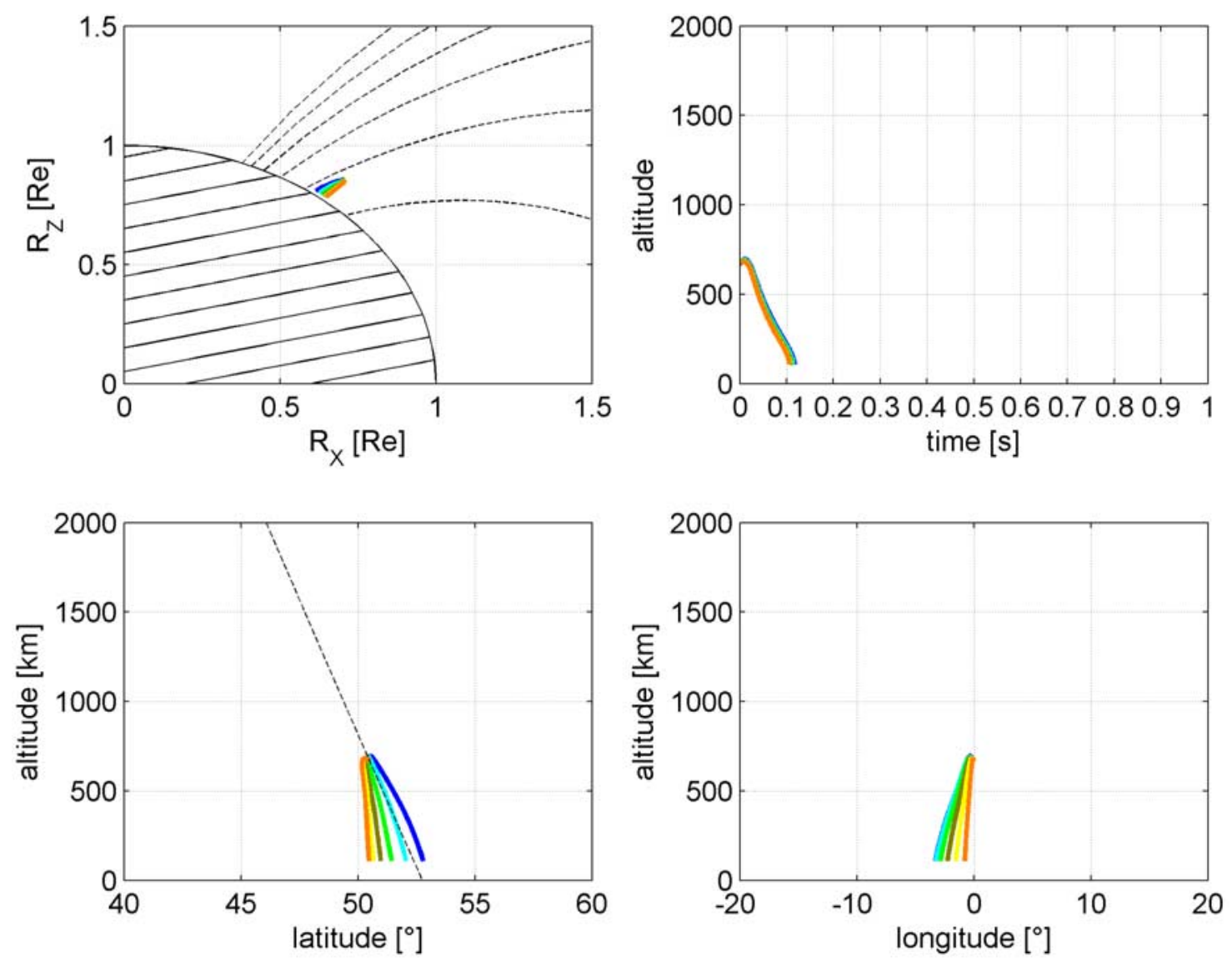

Figure 6. Results of the ray tracing for raypaths started at the point of the satellite with the initial angle $\theta=96^{\circ}$ (third trace) and various initial values of $\varphi$, which are color-coded as follows: $\varphi=-60$ (blue), $\varphi=$ $-80^{\circ}$ (cyan), $\varphi=-100^{\circ}$ (green), $\varphi=-120^{\circ}$ (olive), $\varphi=-140^{\circ}$ (yellow), and $\varphi=-160^{\circ}$ (orange). All coordinates are geomagnetic. The initial longitude of the satellite is supposed to be 0 . See section 3 for more details.

Table 2. Results of the Ray Tracing for the Typical Values of $\theta$ and $\varphi$ Observed at the Satellite for Different Traces $^{\mathrm{a}}$

\begin{tabular}{|c|c|c|c|c|c|}
\hline Parameter & Trace1_u & Trace2_Ld & Trace2_Td & Trace3_Lu & Trace3_Td \\
\hline$\theta$ s $(\operatorname{deg})$ & 160 & $\sim 65$ & $\sim 65$ & $\sim 96$ & $\sim 83$ \\
\hline$\varphi \_s(\operatorname{deg})$ & $\sim-70$ & $\sim 0$ & $\sim-140$ & $\sim-135$ & $\sim-120$ \\
\hline dmlat 0 (deg) & -0.11 & -2.6 & 3.8 & 1.39 & 0.94 \\
\hline dlong_0 (deg) & 0.64 & 0 & 6.8 & 2.31 & 3.4 \\
\hline$\theta 0$ (deg) & 160 & 140.3 & 162.8 & 161.2 & 155.4 \\
\hline$\varphi 0$ (deg) & -10.8 & 0 & -37.9 & -79.9 & -61.4 \\
\hline ts 0 (s) & 0.08 & 0.13 & 0.161 & 0.1 & 0.12 \\
\hline$\alpha \_0(\operatorname{deg})$ & 4.1 & 16.3 & 12 & 25 & 23 \\
\hline dmlat 1 (deg) & no reflection & 1.7 & -0.84 & -1.13 & -0.61 \\
\hline dlong 1 (deg) & no reflection & 0 & -1.2 & -1.74 & -1.92 \\
\hline$\theta \quad 1$ (deg) & no reflection & 3.9 & 32 & 42.3 & 37.8 \\
\hline$\varphi-1(\mathrm{deg})$ & no reflection & 180 & -163 & -157 & -148 \\
\hline ts $1(\mathrm{~s})$ & no reflection & 0.09 & 0.08 & 0.11 & 0.09 \\
\hline$\alpha 1(\mathrm{deg})$ & no reflection & 16.4 & 12.5 & 23.2 & 22 \\
\hline alt_R (km) & no reflection & 776 & 913 & 685 & 704 \\
\hline
\end{tabular}

${ }^{\mathrm{a}}$ Here $\theta s$ and $\varphi s$ represent values of $\theta$ and $\varphi$ observed at the satellite. The name Trace $1 \mathrm{u}$ is used for the upgoing first trace; Trace2_Ld and Trace2_Td are for the leading and trailing edge of the downgoing second trace, respectively; Trace $3 \_\mathrm{Lu}$ and Trace3_Td are for the leading upgoing and trailing downgoing edge of the third trace, respectively. The following parameters are listed: dmlat_ 0 and dlong_0 0 are the difference of latitude and longitude, respectively, between the ray and the satellite footprint at $110 \mathrm{~km}$ for the rays to reach the satellite; $\theta_{-} 0$ and $\varphi_{-} 0$ are the corresponding wave - normal angles; $t_{-} \_$is the time of propagation from the base of the ionosphere to the satellite; and $\alpha 0$ is the angle between the vertical direction and the wave vector. The parameters indexed by the number 1 have the same meaning but correspond to the waves that propagated from the satellite to the base of the ionosphere at $110 \mathrm{~km}$. The last parameter, alt R, gives the altitude of the reflection. 


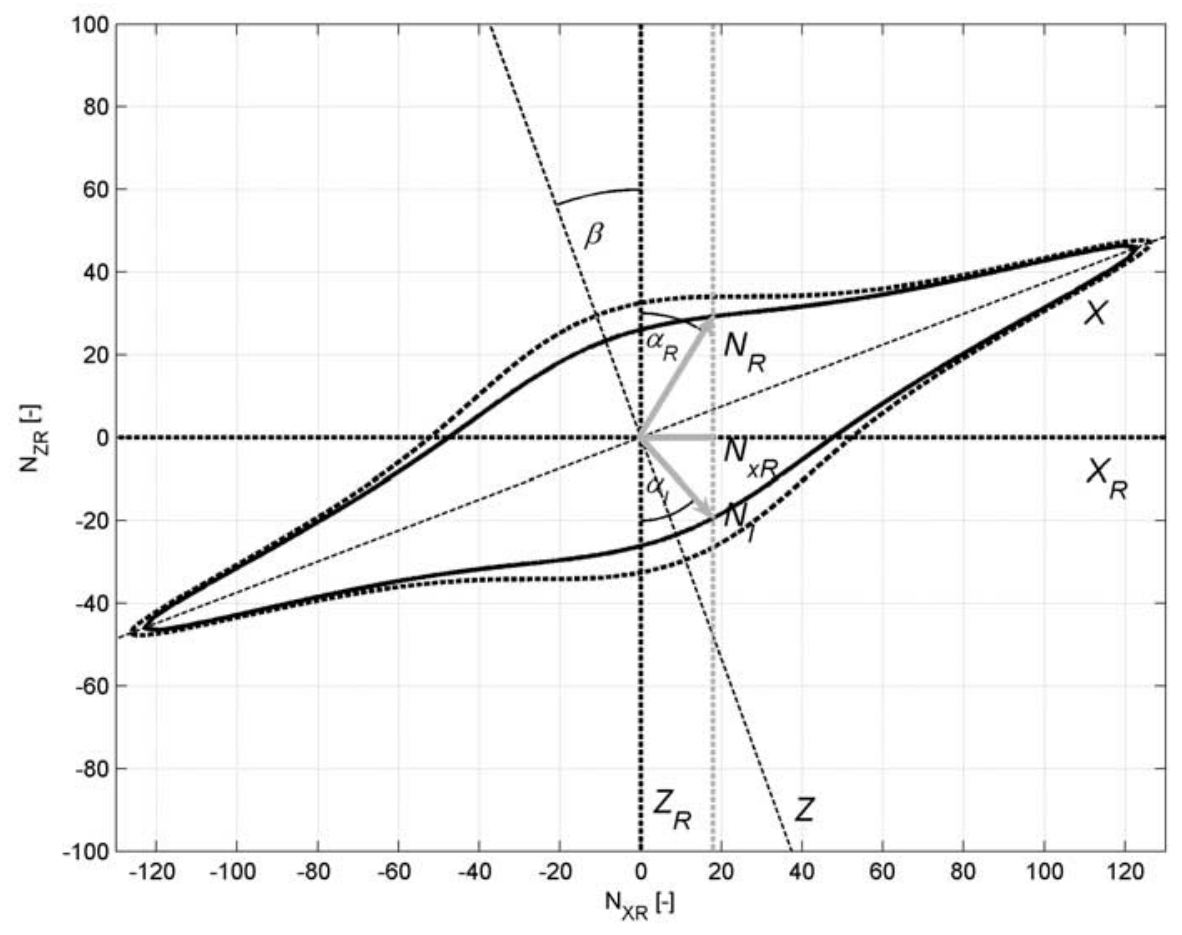

Figure 7. Projection of the surface of the refractive index into the meridian plane and an illustration of the wave-normal directions during the reflection from the horizontally stratified base of the ionosphere. The meaning of the coordinates and symbols is as follows: $Z$ is the direction of the magnetic field, $X$ is the perpendicular axis lying in the meridian plane, $Z_{R}$ is the vertical downward direction, $X_{R}$ is the direction to the north, $\beta$ is the angle between magnetic field and the vertical, $N_{I}$ is the refractive index of the incident wave (projection to meridian plane), $N_{R}$ is the refractive index of the reflected wave, $N_{X R}$ is the $X_{R}$-component of the refractive index $N$, and $\alpha_{I}$ and $\alpha_{R}$ are the angle of incidence and reflection, respectively, with respect to the vertical. According to Snell's law, the $N_{X R}$ and $N_{Y R}$ remain constant during the reflection, so $N_{R} \cos \left(\alpha_{R}\right)=N_{I} \cos \left(\alpha_{I}\right)$. Note that the $N_{y}$ component is not seen because it is perpendicular to the figure plane. The solid curves represent the refractive index surface in the case when $N_{y}$ is nonzero, and the dashed curve indicates the cross section of refraction surface in the case when $N_{y}=$ 0 . The gray dashed straight line indicates the vertical at the points of the refractive index vector. The values of $N_{x}$ and $N_{y}$ (not seen) were exaggerated for the purpose of the illustration.

[23] It is interesting to note that the initial angles $\theta_{-} 0$ for various traces are similar at the base of the ionosphere, though the corresponding trajectories are remarkably different. The first trace starts almost vertically. Nevertheless, the deviation from vertical direction is relatively low also for the next traces; the maximum deviation of $25^{\circ}$ was found for the leading edge of the third trace. It is obvious that the raypaths may exhibit a divergent behavior in dependence on the initial conditions. A discussion of the ray tracing results and their comparison with the observations is presented in section 4 . We will also investigate where the third trace propagated from before it experienced the reflection from the lower boundary of the ionosphere.

\section{Comparing and Discussing the Observations With the Results of the Ray Tracing Simulations}

\subsection{Formation of Components of SP Whistler and the} Bottom Reflection

[24] The ray tracing results indicate that the reflected components (echoes) of a SP whistler are not formed by the waves corresponding to the first trace (component) observed on the satellite. Though we cannot exclude that there is a certain spread of wave-normal angles in the first trace, the main energy is formed by waves propagating almost antiparallel to magnetic field $\left(\theta=160^{\circ}\right)$, which do not reflect and escape to the opposite hemisphere. The multicomponent measurements of the wave polarization of the first trace indicate that the waves are planar; the spread of wave-normal angles is relatively low in this case. It is obvious that the subsequent components (echoes) are formed by the waves that entered into the ionosphere in other regions and at other wave-normal angles. We have already presented the backward ray tracing for the first, second, and third trace. In that respect it is interesting to follow the raypath of the third trace before it potentially reflected from the lower boundary of the ionosphere. We will suppose that the lower boundary is horizontal. Therefore, according to the Snell's law, the horizontal component $N_{H}$ of the refractive index $N$ is preserved during the reflection. Note that if $N_{H}>1$, the waves are totally reflected upward because the maximum of $N$ in the neutral atmosphere (free space) is 1 .

[25] Figure 7 shows, analogously to Poeverlein construction, the projection of the surface of the refractive index $N$ into the meridian plane. The $Z$ axis represents the direction 
of the ambient magnetic field. The vertical downward direction is represented by the $Z_{R}$ axis and the direction to the north in the horizontal plane by the $X_{R}$ axis. Between the components of the refractive index in these two coordinate systems the following relations hold:

$$
\begin{gathered}
N_{X R}=N_{X} \cos \beta+N_{z} \sin \beta, \\
N_{Y R}=N_{Y}, \\
N_{Z R}=-N_{X} \sin \beta+N_{z} \cos \beta,
\end{gathered}
$$

where

$$
\begin{gathered}
N_{X}=N \sin \theta \cdot \cos \phi, \\
N_{Y}=N \sin \theta \cdot \sin \phi, \\
N_{Z}=N \cos \theta,
\end{gathered}
$$

$\beta$ is the angle between the vertical direction and magnetic field. Supposing a dipole magnetic field, the magnetic field lines are described by the equation (9)

$$
R=L \cos ^{2}\left(\lambda_{M}\right)
$$

where $R$ is the distance from the center of Earth in Earth radii, $\lambda_{M}$ is the magnetic latitude (mlat), and $L$ is the distance of the magnetic field line from the center of Earth in the magnetic equatorial plane. Using (9), the angle $\beta$ can be calculated as follows:

$$
\operatorname{tg} \beta=\frac{-R \cdot d \lambda_{M}}{d R}=\frac{-L \cos ^{2} \lambda_{M} \cdot d \lambda_{M}}{-2 L \cos \lambda_{M} \cdot \sin \lambda_{M} \cdot d \lambda_{M}}=\frac{1}{2} \operatorname{ctg} \lambda_{M}
$$

Snell's law requires that both the horizontal components $N_{X R}$ and $N_{Y R}$ are preserved. That means that also the azimuthal angle $\psi$ defined by (11) is conserved:

$$
\operatorname{tg} \psi=\frac{N_{Y R}}{N_{X R}} .
$$

To follow the third trace backward, before its reflection, we have to find how the angles $\theta$ and $\varphi$ change during the reflection at the lower boundary of the ionosphere. The angles $\theta$ and $\varphi$ can be found by the following method: We stop the ray tracing at an altitude of $110 \mathrm{~km}$. Using equations (3)-(5) we calculate the $N_{X R I}$ and $N_{Y R I}$ components of $N$ for the incident backward traced ray. Note that the angle $\theta$ for the incident ray lies in the interval from $\theta \leq$ $\theta<90^{\circ}$ whereas the angle $\theta$ for the searched reflected ray lies in the interval $90^{\circ}<\theta \leq 180^{\circ}$ on the Northern Hemisphere. Using the dispersion relation for cold plasmas and the equations (3)-(8), we search for such angles $\theta$ and $\varphi$ from the interval $90^{\circ}<\theta \leq 180^{\circ}$ and $-180^{\circ} \leq \varphi<180^{\circ}$, respectively, which satisfy $N_{X R}=N_{X R I}$ and $N_{Y R}=N_{Y R I}$. Because the dependence of $N$ on $\theta$ through the dispersion relation is relatively complicated, we used a numerical approach. We searched for such $\theta$ and $\varphi$ for which the quantity $\left(N_{X R}-N_{X R I}\right)^{2}+\left(N_{Y R}-N_{Y R I}\right)^{2}$ is minimum. The angle $\theta$ was changed with the $0.1^{\circ}$ step and the angle $\varphi$ with the $0.5^{\circ}$ step. The angles $\theta$ and $\varphi$ found by this way were used as the initial conditions for the next run of the ray tracing procedure.

[26] The results are presented in Figure 8. The raypath of the third trace is drawn by the gray solid line for the leading edge and by the gray dashed line for the trailing edge. The black curves represent the backward paths of waves with the initial conditions corresponding to those which can be observed in the second trace. Note that it is obvious from Figure 2 that the second trace is formed by multiple rays; there is a noise in the values of $\theta$ and especially $\varphi$. Worth noticing is the fact that the regions where the trajectories of the second and third trace (before its reflections) started can overlap.

[27] The results of ray tracing analysis presented in Figure 8 lead to a conclusion that a region exists in which the waves penetrate the base of the ionosphere with a large spread of wave-normal angles. This region, which we will call a spread region, is different from the region where the first trace entered. Some of the waves that entered in the spread region reach the observation point, the satellite, and some of them reflect elsewhere. The time delay for waves that reach the satellite depends on the number of reflection that these waves experience and on the extent of the spread region. For example, the waves of the second trace experienced one upper reflection, whereas the waves of the third trace experienced one upper reflection, one lower reflection, and the other upper reflection takes place during the observation.

[28] Obviously, we cannot exclude that more spread regions exist. The spread in angle $\varphi$ observed for the second trace indicates that the spread region from which the second trace propagates is much larger than the spread region from which the third trace propagates. Note that it is also possible that the waves with the quasi-vertical wave vectors enter in the spread region, but these propagate to the other hemisphere and do not reach the satellite.

[29] Our observation of wave-normal angles and related ray tracing results are thus partially inconsistent with the hypothesis proposed by Kimura [1966], who supposed that all the components of a SP whistler propagate along one specific path. Note that Kimura [1966] had no measurements of the wave-normal angles on the satellite. To achieve one specific path in his ray tracing studies, he introduced horizontal gradients in the ionosphere. Our ray tracing study on the basis of the real measurement of the wave-normal angles showed that different components of SP whistler propagate along different trajectories and reach the satellite with different wave-normal angles after they have experienced a different number of reflections. The different trajectories result not only from the different entering points into the ionosphere, but mainly from the different initial wave-normal angles in the lower ionosphere where the waves undergo a spread of wave-normal angles. This spread of the initial wave-normal angles is the primary cause that a part of whistler energy is trapped below the protonosphere. The large-scale horizontal gradients are not important for the observation of the subsequent components. The propa- 

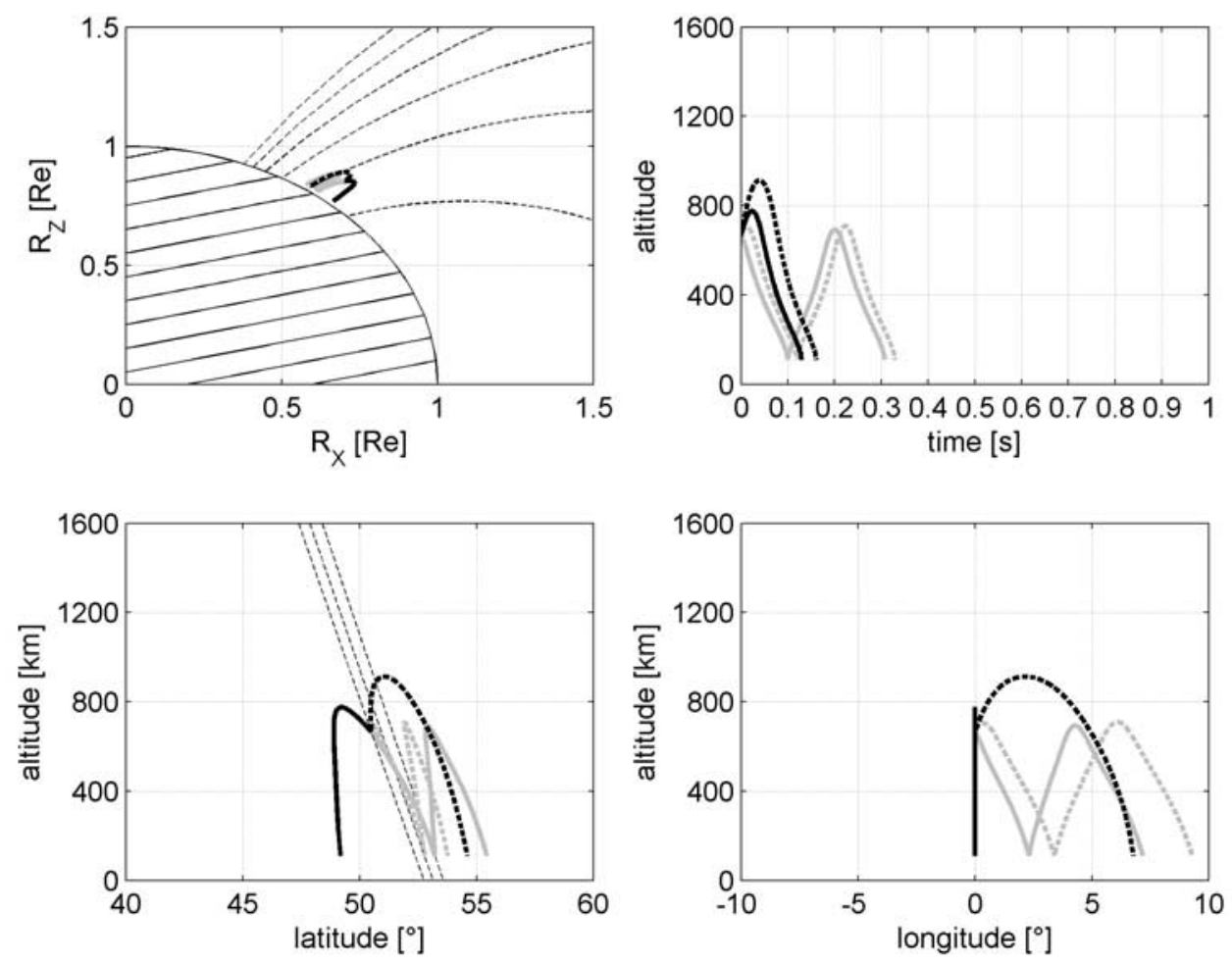

Figure 8. Composite results of the backward ray tracing for the second trace (represented by the black curves) and for the third trace (represented by the dashed curves). The initial conditions were as follows: $\theta=115^{\circ}$ and $\varphi=180^{\circ}$ for the black solid line, $\theta=115^{\circ}$ and $\varphi=40^{\circ}$ for the black dashed line, $\theta=84^{\circ}$ and $\varphi=45^{\circ}$ for the gray solid line, and $\theta=97^{\circ}$ and $\varphi=60^{\circ}$ for the gray dashed line. The reflection from the base of the ionosphere was treated as described in section 4 . Note that the waves can arrive to the observation point after a different number of reflections.

gation at relatively large horizontal distances from the penetration region (spread region) and also the propagation perpendicular to the meridian plane is observed. This explanation is also partially different from the hypothesis that was put forward by Raghuram [1975] who suggested that the different components of SP whistlers are observed owing to the waves traveling along different paths and exhibiting different number of reflections. Raghuram [1975], however, only considered the propagation in the meridian plane and that the different trajectories results from the waves entering at different latitudes. Raghuram [1975] did not mention the important fact that different components are formed by the waves propagating at different wavenormal angles owing to the initial spread of wave-normal angles. Our analysis and observations show that apart from the different entering points, the different raypaths are a consequence of the different wave-normal angles and that propagation across the meridian planes is important in most cases. We have also shown that different components of the SP whistlers reflect at different altitudes.

[30] As for the mechanism which causes the initial spread of wave-normal angles, we assume, similar to Kimura [1966], that (1) "pinholes" in a sporadic $E$ layer and/or (2) density irregularities and subsequent scattering on smallscale irregularities can be responsible for that. We observed a semitransparent sporadic $E$ layer by the digisonde located in Pruhonice $\left(49^{\circ} 59^{\prime} \mathrm{N}, 14^{\circ} 32^{\prime} \mathrm{E}\right)$ at 2115 on 9 July 2007. Note that the sampling rate of the digisonde was $15 \mathrm{~min}$.
Therefore, we do not have the ionogram from exactly the same time. The role of sporadic $E$ layer and density irregularities in the $E$ layer in the wave-normal scattering is worth further investigation.

\subsection{Upper Reflection and the Role of the LHR Frequency}

[31] Kimura [1966] showed that the effect of ions on the whistler mode propagation is important only for waves at frequency $f$ lower than the LHR frequency $f_{L H}\left(f<f_{L H}\right)$. In the case of $f>f_{L H}$, the surface of refractive index is open and the forward and backward propagating modes (with respect to magnetic field line) are separated. A resonance cone determines the maximum angle $\theta$ and the dispersion is similar to the case when the ions are neglected. On the other hand, in the case of $f<f_{L H}$, the surface of refractive index is closed, the value of $\theta$ is not limited, and the backward and forward propagating modes are not separated. At $f=f_{L H}$, the refractive index $N$ goes to infinity for perpendicularly propagating waves $\left(\theta=90^{\circ}\right)$. Figure 9 presents the shapes of the surfaces of the refractive indexes for different ratios $f / f_{L H}$ and the case $f<f_{L H}$. Note that the surface is always symmetric with respect to the magnetic field. The direction of the group velocity is given by the normal to this surface at a given point. It is obvious that the reversal of the group velocity, the reversal of the parallel component respectively, can only occur on the closed surface. In other words, a necessary condition for the backward refraction is $f<f_{L H}$. 


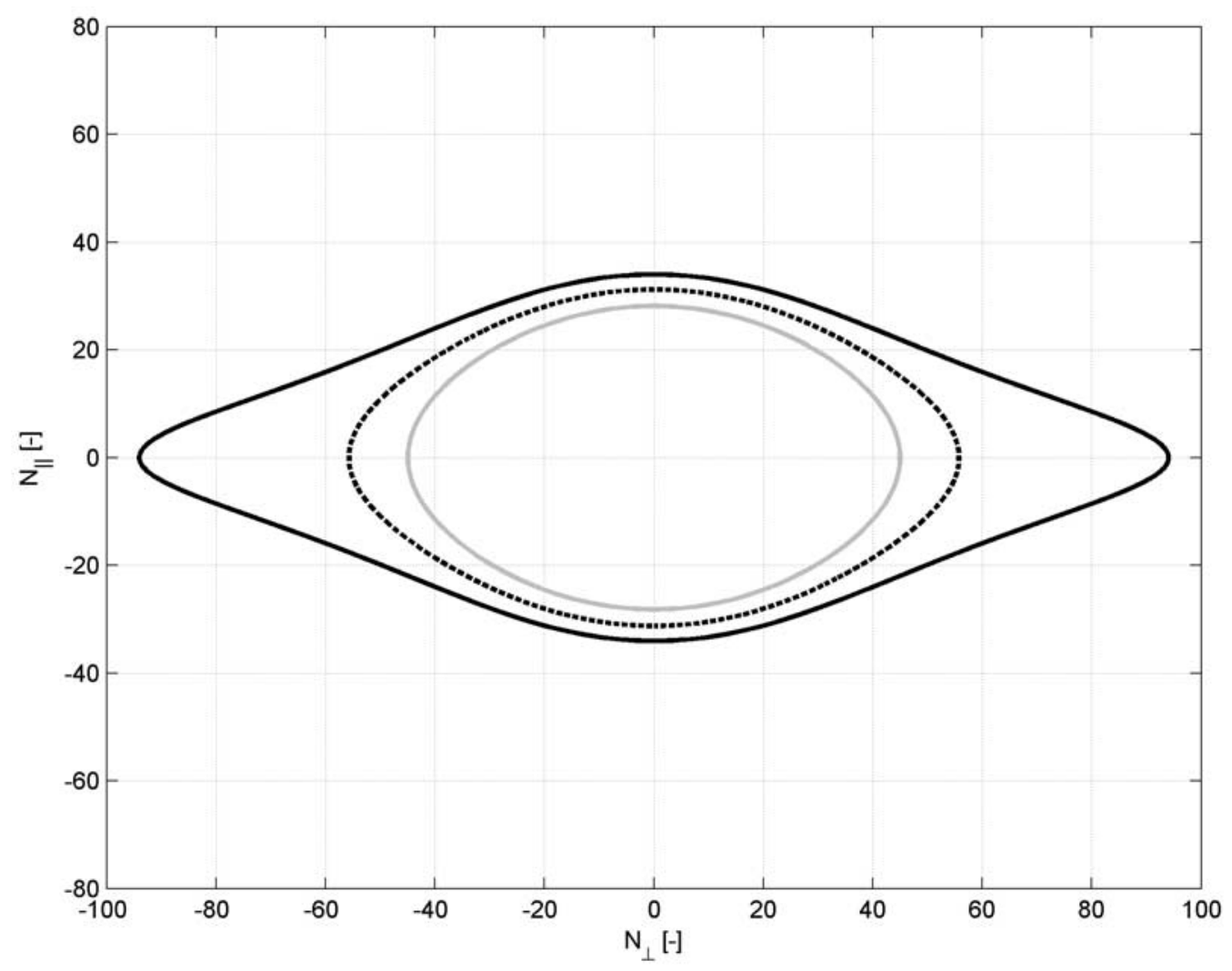

Figure 9. Surface of wave refractive index $N$ for $f=800 \mathrm{~Hz}$ and various LHR frequencies and ratios of ion species, respectively. The plasma frequency and electron frequency are $1 \mathrm{MHz}$. The gray curve stands for plasma containing $90 \%$ of $\mathrm{H}^{+}$and $10 \%$ of $\mathrm{O}^{+}\left(f_{L H}=15.7 \mathrm{kHz}\right)$. The black dashed curve is used for the composition $50 \%$ of $\mathrm{H}^{+}$and $50 \%$ of $\mathrm{O}^{+}\left(f_{L H}=12.0 \mathrm{kHz}\right)$. The black solid curve stands for the composition $10 \%$ of $\mathrm{H}^{+}$and $90 \%$ of $\mathrm{O}^{+}\left(f_{L H}=6.5 \mathrm{kHz}\right)$.

Figure 9 also demonstrates that the transverse waves propagating with $\theta$ close to $90^{\circ}$ are very sensitive to the LHR frequency $f_{L H}$. For a transverse wave of a fixed frequency $f$, $N$ decreases with increasing $f_{L H}$. If the wave is sufficiently transverse and the increase of $f_{L H}$ is large enough, then the decrease of $N$ causes that the wave cannot propagate further and the group velocity is reversed.

[32] Indeed, from the ray tracing results, it follows that the LHR frequency always increases along the wave path before the reflection takes place and the maximum altitude of the upper reflection corresponds to the maximum of LHR frequency above the satellite. This can be seen in Figure 10, where the LHR frequency along the raypaths as a function of altitude is displayed in the upper left plot. Figure 10 also shows the evolution of wave-normal angles in both coordinate systems (see section 4.1) and the evolution of $N$ and its components along the ray trajectories. We conclude that the upper reflection can be viewed as the LHR reflection. The principle of this reflection is basically the same as in the case of the reflection which experience magnetospherically reflected (MR) whistlers observed first by Smith and Angerami [1968] on the OGO 1 and 3 satellites. Nevertheless, there is a significant difference between these two cases. In the case of MR whistlers, the waves propagate from the opposite hemisphere and their frequency is higher than the local LHR frequency before they approach the reflection region. If a wave propagates from a region where
$f>f_{L H}$ and its wave vector is close to the resonance cone (the transverse component of $N \rightarrow \propto$ ), then the reflection occurs almost immediately at a frequency $f \approx f_{L H}$ since the wave cannot propagate into a medium where the transverse component of $N$ is finite. These waves are also called quasielectrostatic waves because the electric field oscillates practically in the direction of $k$ and their magnetic component is negligible.

[33] The LHR reflection of quasi-electrostatic waves can also cause a visible cutoff in the spectrograms. The reason for the presence of quasi-electrostatic waves can often be a scattering of whistler mode waves on small-scale density irregularities (see Bell and Ngo [1990] or Shklyar and Nagano [1998] and references therein). An example of LHR cutoff and LHR emissions generated by scattering of downgoing quasi-electrostatic waves can be seen in the spectrogram in Figure 1 at frequencies from $\sim 11 \mathrm{kHz}$ to $\sim 12 \mathrm{kHz}$. The cutoff frequency corresponds to the maximum of LHR frequency which is located above the satellite in our case. Note that the maximum of LHR is relatively close to the satellite and that this value corresponds reasonably well with the values obtained in the ray tracing modeling. Similar quasi-electrostatic emissions just above the LHR cutoff frequency can also be generated by upward propagating waves as was reported, e.g., by Bell et al. [1993, Figure 2]. They observed the LHR emissions on ISIS 2 satellite at the altitude of $\sim 1400 \mathrm{~km}$. Note that in their 

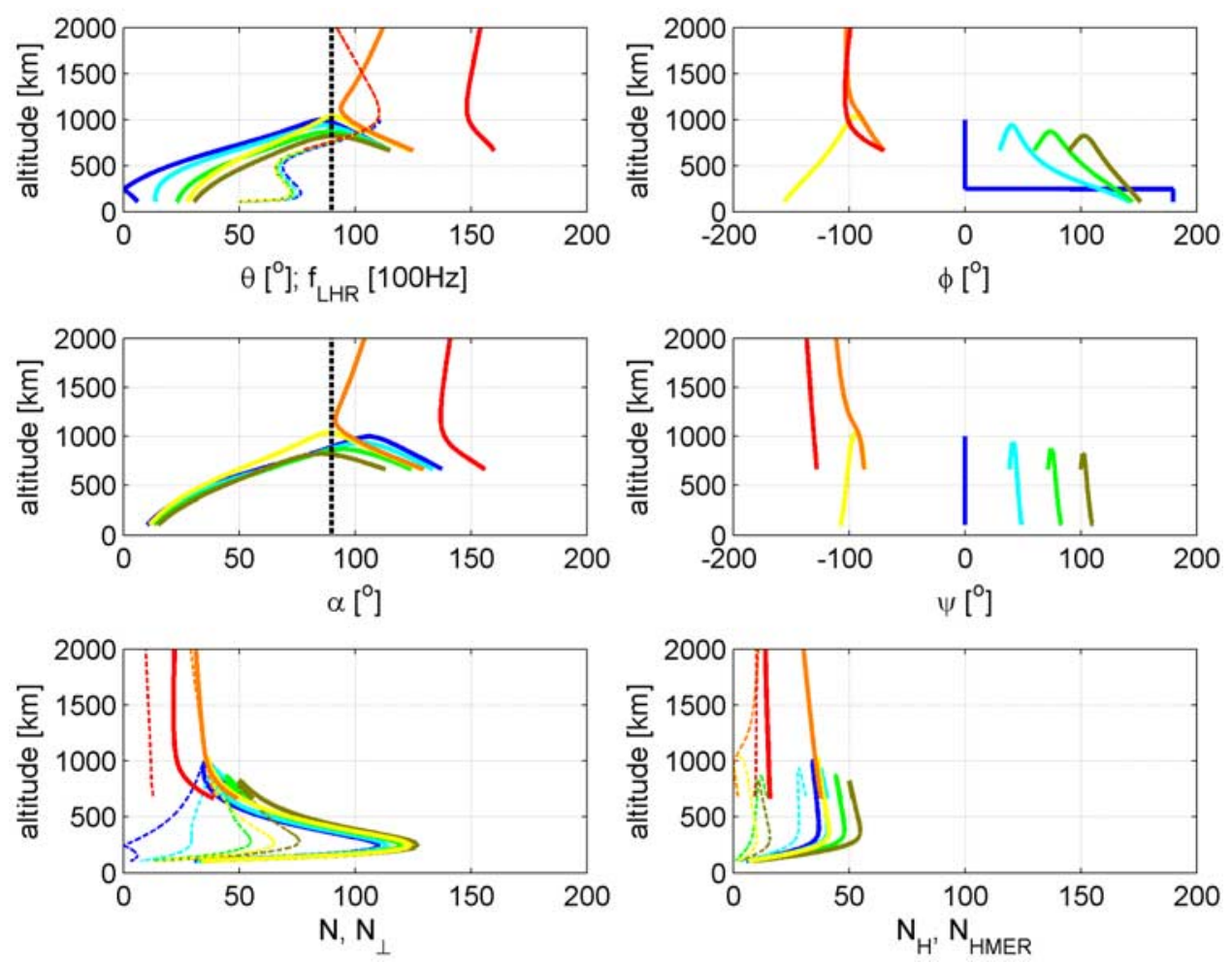

Figure 10. Composite results of the ray tracing demonstrating that the reflection always takes place below the maximum of LHR frequency. The LHR frequency as a function of altitude along the wave paths is indicated by the dashed lines in the upper left plot and is given in $100 \mathrm{~Hz}$ (multiply the numbers on horizontal axis by 100 to get the LHR frequency in Hz; the numbers on horizontal axis simultaneously represent the values of the angle $\theta$ ). Note that the LHR frequency has to increase along the wave path so that the reflection could occur. The individual plots present the evolution of the wave-normal angles $\theta$ and $\varphi$, of the angle $\alpha$ between the wave vector and the vertical direction ( 0 for downward propagation waves), its azimuth $\psi$, the wave refractive index $N$, its perpendicular component $N_{\perp}$ (dashed), its horizontal component $N_{H}$, and its horizontal component projected into meridian plane $N_{H M E R}=N_{X R}$ (dashed) as a function of the altitude. The blue, cyan, green, and olive lines correspond to trajectories in Figure 5. The yellow, orange, and red lines correspond to trajectories in Figure 4.

case, the spacecraft was located, most probably, just above the maximum of LHR frequency (see the map of LHR frequency in Figure 11 discussed at the end of this section), whereas in our case, the satellite was below the maximum of LHR frequency. Note also that in their case, the intensity of LHR emissions increased during the fractional hop whistler $(0+$ whistler) occurrence, whereas in our case, the intensity of LHR emissions increased when a diffusive whistler with a dispersion corresponding to the propagation from the opposite hemisphere was observed ( $\sim 6 \mathrm{~s}$ from the beginning on the spectrogram in Figure 1).

[34] The reflection can also occur for downgoing waves which are oblique, but not close to, the resonance cone. In this case, the reflection takes place at frequencies which are sufficiently lower than the local LHR frequency, providing that these waves experience an increase of the LHR frequency along their trajectory. Such a kind of reflection is often observed in the case of $\mathrm{Nu}$ whistlers [Shklyar et al., 2004], which are MR whistlers observed close to the region of reflection. Note that the important difference between the reflection that experience the $\mathrm{Nu}(\mathrm{MR})$ whistlers and that undergo the SP whistlers is that the SP whistlers propagate all the time in the region where their frequency is lower than the local LHR frequency and that the LHR reflection occurs for the upgoing waves.

[35] Another interesting similarity between the generalized LHR reflection for the downward and upward propagating waves is the divergent behavior for the waves propagating close to the critical conditions of the reflection. For the upgoing waves, the divergence can be observed, e.g., in Figure 4. For the downgoing waves, it was studied, e.g., by Chum and Santolik [2005].

[36] It is worth mentioning that the conclusion that a LHR frequency profile above the satellite plays a crucial role for the upper reflection of SP whistlers is consistent with the fact that SP whistlers are nighttime phenomenon [Carpenter et al., 1964]. Figure 11 presents the LHR frequency as a function of altitude and magnetic local time calculated for the L-shell of observation and for a moderate solar activity using the model of relative ion densities and electron density in the topside ionosphere described by Trísková et al. [2003, 2006] and Truhlik et al. [2004]. This model is a 


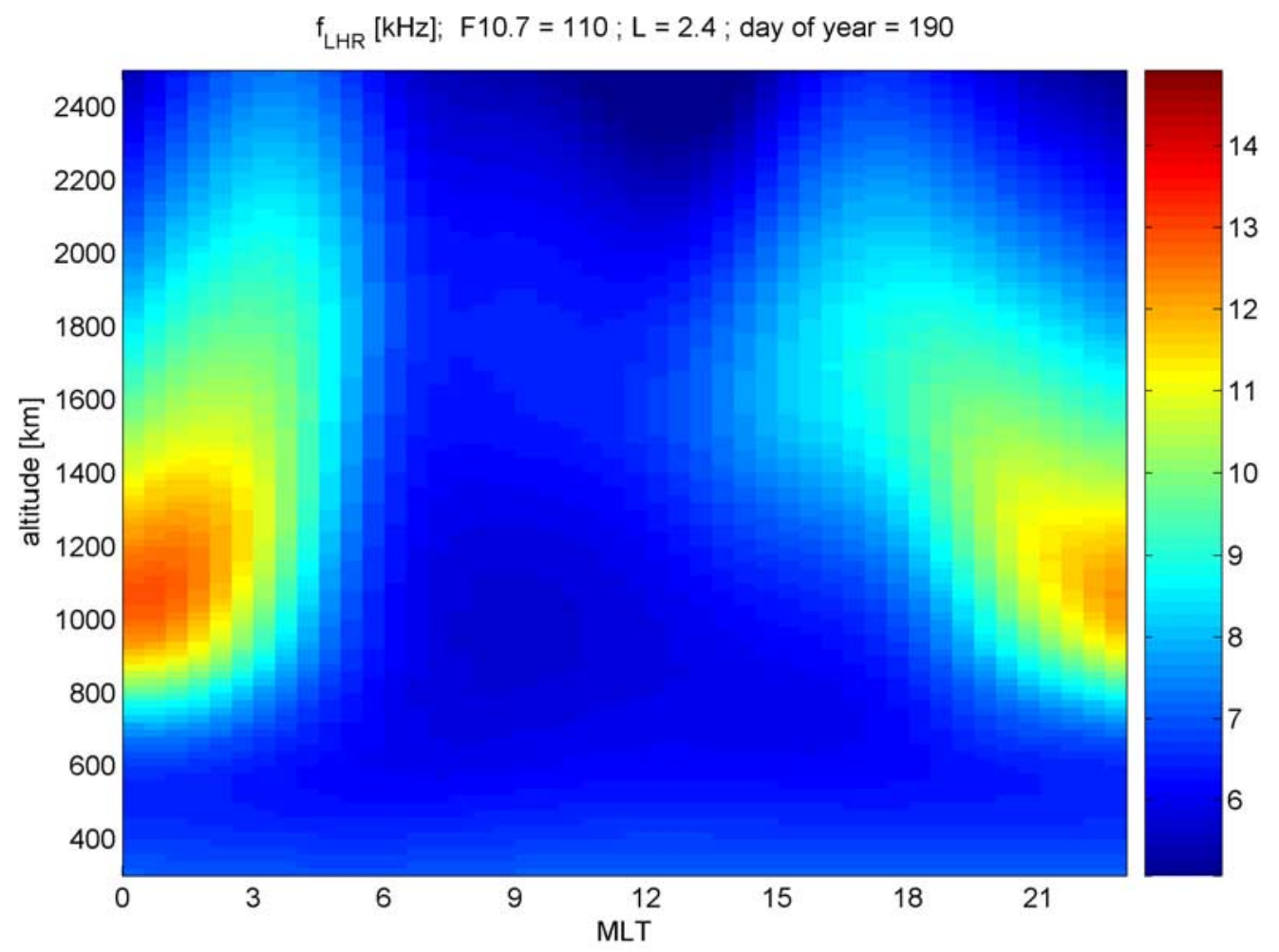

Figure 11. A map of LHR frequency as a function of altitude and magnetic local time for the $\mathrm{L}=2.4$, 200th day in a year and moderate solar activity on the basis of the TTS model. See section 4.2 for more details.

part of international reference ionosphere (IRI) and is referenced as the TTS model. The significant increase of LHR frequency at altitudes above the DEMETER orbit in the nighttime is obvious.

\subsection{Time Delays Between Components and Sensitivity to Plasma Density and Magnetic Field Model}

[37] The average time delay between the second and third component yielded by the ray tracing in Figure 8 is $\sim 0.17 \mathrm{~s}$. The observed time delay seems to be a bit larger, about $0.18 \mathrm{~s}$ between the centers of the traces. However, we cannot exclude that the density measured by the satellite is a bit underestimated or that our diffusive equilibrium density model on the basis of fixed temperature measured on DEMETER is not exact. The effective temperature of this model is probably lower than that observed at the spacecraft altitude; see Santolik et al. [2009] for more details concerning this problem. Note that the IRI model gives higher values of electron density, almost $30,000 \mathrm{~cm}^{-3}$ at the satellite altitude. Moreover, the highest density along the backward traced ray of the first trace was $175,000 \mathrm{~cm}^{-3}$ at the altitude $239 \mathrm{~km}$. That density corresponds to the maximum plasma frequency of $3.76 \mathrm{MHz}$, whereas the critical frequency $f_{o} F_{2}$ of the ionosphere measured by digisonde at Pruhonice $\left(49^{\circ} 59^{\prime} \mathrm{N}, 14^{\circ} 32^{\prime} \mathrm{E}\right)$, located approximately between the causative lightning and the satellite footprint was $\sim 5.2 \mathrm{MHz}$. The critical frequency $f_{o} F_{2}$ of $\sim 5.2 \mathrm{MHz}$ is also obtained by IRI. The ray tracing simulation confirms that the time of propagation increases with density, whereas the raypaths change negligibly, provided that we use the same density model otherwise. Therefore, our results are qualitatively independent on the density used. Note that the density can also influence the critical angles and trajectories corresponding to the critical angles because the maximum of LHR depends on the density.

[38] We have also tested the sensitivity of our results to magnetic field model using the IGRF magnetic field model and the same ray tracing procedure as Santolik et al. [2009]. We have found small changes in the raypaths, times of propagation, and LHR frequencies. For example, the maximum of LHR frequency above the satellite is $10.94 \mathrm{kHz}$ for IGRF model (11.04 kHz for the dipole approximation). The first trace propagated to the other hemisphere. The altitude of reflection of the second component changed from $776 \mathrm{~km}$ to $779 \mathrm{~km}$ for the leading edge and from $913 \mathrm{~km}$ to $925 \mathrm{~km}$ for the trailing edge, respectively. The difference in propagation time was $\sim 0.002 \mathrm{~s}$ for these two models, and the difference in longitude of the backward traced trajectories at the base of the ionosphere was $\sim 0.5^{\circ}$. It is obvious that our qualitative interpretation is independent on the magnetic field model used.

\subsection{Dispersion}

[39] Carpenter et al. [1964] pointed out that the components of the SP whistlers approximately follow the dispersion law $t=D f^{-1 / 2}$ with a small deviation at lower frequencies. In Figure 12, we show that the case studied fits this dispersion law rather well. On the zoomed spectrogram containing the SP whistler analyzed, we overlaid the curves corresponding to the dispersions $D=2.5,5,10,15$, 


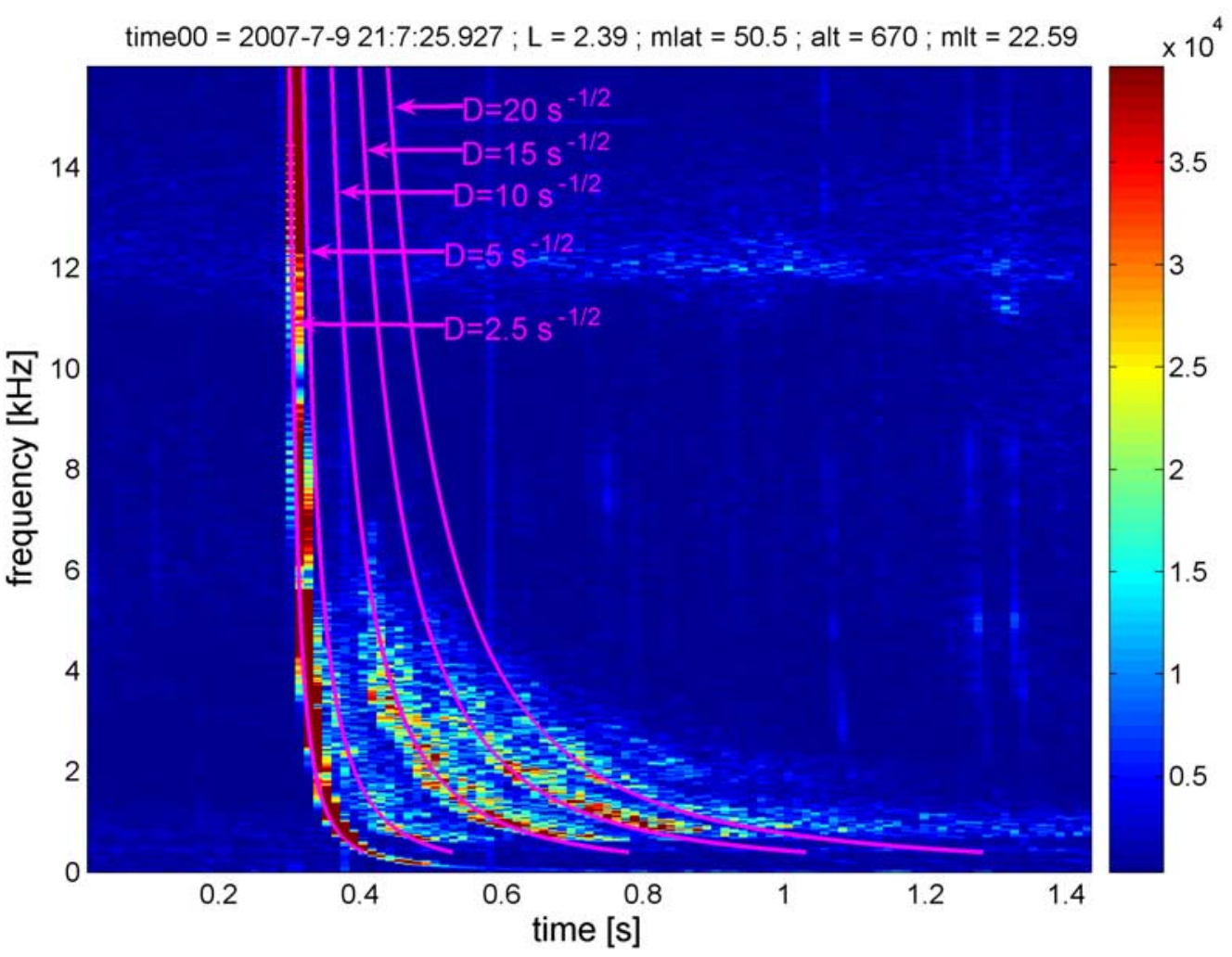

Figure 12. A detailed amplitude spectrogram of the SP whistler analyzed and curves corresponding to the dispersions $D=2.5,5,10,15$, and $20 \mathrm{~s}^{1 / 2}$.

and $20 \mathrm{~s}^{1 / 2}$ which approximate the individual components of the SP whistler. We have got similar results also for other cases; e.g., the dispersions of the SP whistler components observed on 25 June 2005 at 2053:55.5 UT could be approximated by values $D=2.8,5.6,11.2$, and $17 \mathrm{~s}^{1 / 2}$.

[40] Next, we will compare the "observed" value of dispersion with the results of the ray tracing analysis. So far we have presented the ray tracing simulations at a frequency $f=800 \mathrm{~Hz}$. To compare the simulated times of propagation at different frequencies with the observed dispersion, we have also performed the ray tracing simulation at the frequency $f=1200 \mathrm{~Hz}$. For the second component of the SP whistler and wave packets of frequencies $f=$ 800 and $1200 \mathrm{~Hz}$ propagating approximately along the same trajectories, we get the simulated times of propagation $0.161 \mathrm{~s}$ and $0.135 \mathrm{~s}$, respectively. The dispersion $D=5$, which approximates the observed trace of the second component in the spectrogram, corresponds to the propagation times of $0.176 \mathrm{~s}$ and $0.144 \mathrm{~s}$ at frequencies $f=800$ and $1200 \mathrm{~Hz}$, respectively. Thus, the observed and simulated times of propagation are in a reasonable agreement. Note that the simulated dispersion is a bit smaller, but consistently with the smaller time delay between the simulated components of SP whistler. The reason for the smaller values obtained in the simulations is the inaccuracy of the density model used; see the discussion in section 4.3.

[41] The ray tracing simulation also show that the sensitivity of oblique waves on the LHR frequency profile decreases with the increasing frequency. Thus, the lower frequencies are reflected more easily. This is consistent with the results obtained by Kimura [1966] and explanation suggested by Smith [1964].

\section{Conclusions}

[42] We presented a ray tracing analysis of SP whistlers on the basis of the initial values obtained by measurements on board the satellite DEMETER. To find the wave-normal angles at the satellite point, we used a plane wave analysis using three components of magnetic and three components of electric field. The plasma density model used in the ray tracing technique was fitted to the ion relative densities and electron density measured by the satellite.

[43] We conclude that the components of SP whistlers are formed by the waves that propagate in a waveguide formed by the profile of the increasing LHR frequency in the upper ionosphere and the lower boundary of the ionosphere. The waves forming the reflected components (echoes) enter the ionosphere at relatively large horizontal distances with respect to the satellite footprint and during this entry experience a spread of wave-normal angles. Depending on the initial wave-normal angle, these waves experience a different number of reflections before reaching the satellite, thus propagating along different paths and arriving with different time delays. These waves often propagate across the magnetic meridian planes. The causative lightning is usually strong and located at relatively large horizontal distances, almost $1000 \mathrm{~km}$ in the analyzed case.

[44] The maximum possible altitude of the upper reflection corresponds to the maximum of the LHR frequency above the satellite. 
[45] As for the first trace observed of a SP whistler, this enters the ionosphere at relatively small horizontal distances from the satellite footprint. It does not reflect above the satellite and propagates to the opposite hemisphere.

[46] Acknowledgments. This work is based on observations with the electric field experiment ICE and the magnetic field experiment IMSC embarked on DEMETER, which was launched by the Centre National d'Etudes Spatiales. We thank J. J. Berthelier for the DEMETER ICE electric field data. We thank Gerhard Diendorfer and all the member organizations of EUCLID for providing the lightning data and Dalja Buresova for the ionogram from the observatory of Pruhonice. Jaroslav Chum also acknowledges the valuable discussion with D. R. Shklyar related to the role of LHR frequency on whistler mode propagation. We would like to acknowledge grants 205/06/1267 and 205/06/0875 of the Grant Agency of the Czech Republic, grants A300420603 and A301120601 of the Grant Agency of the Academy of Sciences of the Czech Republic, and grant RITA-CT-2006-025969 of LAPBIAT 2.

[47] Amitava Bhattacharjee thanks Vikas Sonwalkar and Dennis Papadopoulos for their assistance in evaluating this paper.

\section{References}

Barrington, R. E., and J. S. Belrose (1963), Preliminary results from the very low frequency receiver aboard Canada's Alouette satellite, Nature, 198(4881), 651-656, doi:10.1038/198651a0.

Bell, T. F., and H. D. Ngo (1990), Electrostatic lower hybrid waves excited by electromagnetic whistler mode waves scattering from planar magnetic field aligned plasma density irregularities, J. Geophys. Res., 95, 149 172, doi:10.1029/JA095iA01p00149.

Bell, T. F., R. A. Helliwell, U. S. Inan, and D. S. Lauben (1993), The heating of suprathermal ions above thunderstorms cells, Geophys. Res. Lett., 20, 1991-1994, doi:10.1029/93GL02242.

Berthelier, J., et al. (2006a), ICE, the electric field experiment on DEMETER, Planet. Space Sci., 54, 456-471, doi:10.1016/j.pss.2005.10.016.

Berthelier, J., M. Godefroy, F. Leblanc, E. Seran, D. Peschard, P. Gilbert, and J. Artru (2006b), IAP, the thermal plasma analyser on DEMETER, Planet. Space Sci., 54, 487-501, doi:10.1016/j.pss.2005.10.018.

Cairo, L., and F. Lefeuvre (1986), Localization of sources of ELF/VLF hiss observed in the magnetosphere: Three-dimensional ray tracing, J. Geophys. Res., 91, 4352-4364, doi:10.1029/JA091iA04p04352.

Carpenter, D. L., N. Dunckel, and J. F. Walkup (1964), A new very low frequency phenomenon: Whistlers trapped below the protonosphere, J. Geophys. Res., 69, 5009-5017, doi:10.1029/JZ069i023p05009.

Cerisier, J. (1970), Propagation perpendiculaire au voisinage de la frequence de la resonance hybride basse, in Plasma Waves in Space and in the Laboratory, vol. 2, pp. 487-521, Edinburgh Univ. Press, Edinburgh, U.K.

Chum, J., and O. Santolik (2005), Propagation of whistler-mode chorus to low altitudes: Divergent ray trajectories and ground accessibility, Ann. Geophys., 23, 3727-3738.

Chum, J., F. Jiricek, O. Santolik, M. Parrot, G. Diendorfer, and J. Fiser (2006), Assigning the causative lightning to the whistlers observed on satellites, Ann. Geophys., 24, 2921-2929.

Eckersley, T. L. (1935), Musical atmospherics, Nature, 135, 104-106, doi:10.1038/135104a0.

Egeland, A., and E. Leer (1970), Low latitude subprotonospheric and ion cyclotron whistlers generated by the same lightning discharge, Planet. Space Sci., 18, 1848-1850, doi:10.1016/0032-0633(70)90022-X.

Gurnett, D. A., and S. D. Shawhan (1965), Ion cyclotron whistlers, J. Geophys. Res., 70, 1665-1688, doi:10.1029/JZ070i007p01665.
Gurnett, D. A., R. S. Mosier, and R. R. Anderson (1971), Color spectrograms of very low frequency Poynting flux data, J. Geophys. Res., 76 , 3022-3033, doi:10.1029/JA076i013p03022.

Kimura, I. (1966), Effects of ions on whistler-mode ray tracing, Radio Sci., $1,269-283$.

Lebreton, J. P., et al. (2006), The ISL Langmuir probe experiment processing onboard DEMETER: Scientific objectives, description and first results, Planet. Space Sci., 54, 472-486, doi:10.1016/j.pss.2005.10.017.

Parrot, M., et al. (2006), The magnetic field experiment IMSC and its data processing onboard DEMETER: Scientific objectives, description an first results, Planet. Space Sci., 54, 512-527, doi:10.1016/j.pss.2005. 10.015 .

Raghuram, R. (1975), A new interpretation of subprotonospheric whistlers characteristics, J. Geophys. Res., 80, 4729-4731, doi:10.1029/ JA080i034p04729.

Russell, C. T., R. L. McPherron, and P. J. Coleman Jr. (1972), Fluctuating magnetic fields in the magnetosphere, Space Sci. Rev., 12, 810-856, doi:10.1007/BF00173072.

Santolik, O., M. Parrot, and F. Lefeuvre (2003), Singular value decomposition methods for wave propagation analysis, Radio Sci., 38(1), 1010 doi:10.1029/2000RS002523.

Santolik, O., F. Nemec, M. Parrot, D. Lagoutte, L. Madrias, and J. J. Berthelier (2006a), Analysis methods for multi-component wave measurements on board the DEMETER spacecraft, Planet. Space Sci., 54 , 512-527, doi:10.1016/j.pss.2005.10.020

Santolik, O., J. Chum, M. Parrot, D. A. Gurnett, J. S. Pickett, and N. Cornilleau-Wehrlin (2006b), Propagation of whistler mode chorus to low altitudes: Spacecraft observations of structured ELF hiss, J. Geophys. Res., 111, A10208, doi:10.1029/2005JA011462.

Santolik, O., M. Parrot, U. S. Inan, D. Buresova, D. A. Gurnett, and J. Chum (2009), Propagation of unducted whistlers from their source lightning: A case study, J. Geophys. Res., doi:10.1029/2008JA013776, in press.

Shklyar, D. R., and I. Nagano (1998), On VLF wave scattering in plasma with density irregularities, J. Geophys. Res., 103, 29,515-29,526, doi:10.1029/98JA02311.

Shklyar, D. R., J. Chum, and F. Jiricek (2004), Characteristic properties of $\mathrm{Nu}$ whistlers as inferred from observations and numerical modelling, Ann. Geophys., 22, 3589-3606.

Smith, R. L. (1964), An explanation of subprotonospheric whistlers, J. Geophys. Res., 69, 5019-5021, doi:10.1029/JZ069i023p05019.

Smith, R. L., and J. J. Angerami (1968), Magnetospheric properties deduced from OGO 1 observations of ducted and nonducted whistlers, J. Geophys. Res., 73, 1-20, doi:10.1029/JA073i001p00001.

Smith, R. L., and N. Brice (1964), Propagation in multicomponent plasmas, J. Geophys. Res., 69, 5029-5040, doi:10.1029/JZ069i023p05029.

Trísková, L., V. Truhlík, and J. Šmilauer (2003), An empirical model of ion composition in the outer ionosphere, Adv. Space Res., 31, 653-663, doi:10.1016/S0273-1177(03)00040-1.

Třísková, L., V. Truhlík, and J. Šmilauer (2006), An empirical topside electron density model for calculation of absolute ion densities in IRI, Adv. Space Res., 37, 928-934, doi:10.1016/j.asr.2005.09.013.

Truhlík, V., L. Tř́sková, and J. Šmilauer (2004), New advances in empirical modelling of ion composition in the outer ionosphere, Adv. Space Res., 33, 844-849, doi:10.1016/j.asr.2003.06.006.

J. Chum, Department of Upper Atmosphere, Institute of Atmospheric Physics, Bocni II/1401, Prague 14131, Czech Republic. (jachu@ufa.cas.cz) M. Parrot, Laboratoire de Physique et Chimie de l'Environnement, Centre National de la Recherche Scientifique, 3A Avenue de la Recherche, F-45071 Orleans, France.

O. Santolik, Department of Space Physics, Institute of Atmospheric Physics, Bocni II/1401, Prague 14131, Czech Republic. 